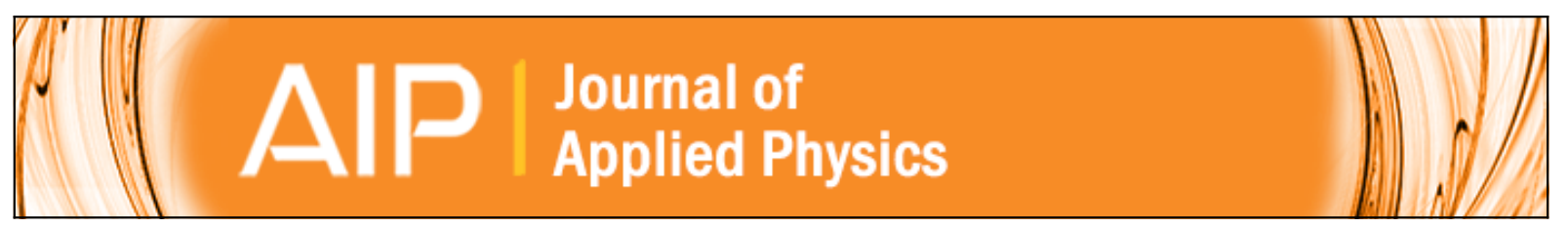

\title{
Examining the temporal evolution of hypervelocity impact phenomena via high-speed imaging and ultraviolet-visible emission spectroscopy
}

J. D. Tandy, J. M. Mihaly, M. A. Adams, and A. J. Rosakis

Citation: Journal of Applied Physics 116, 034901 (2014); doi: 10.1063/1.4890230

View online: http://dx.doi.org/10.1063/1.4890230

View Table of Contents: http://scitation.aip.org/content/aip/journal/jap/116/3?ver=pdfcov

Published by the AIP Publishing

\section{Articles you may be interested in}

Systematic approach to using isentropic stress reverberation techniques in approximating equation of state

Rev. Sci. Instrum. 86, 033908 (2015); 10.1063/1.4914023

Fragmentation of brittle plates by localized impact

Appl. Phys. Lett. 105, 124102 (2014); 10.1063/1.4896773

A model for debris clouds produced by impact of hypervelocity projectiles on multiplate structures Appl. Phys. Lett. 93, 211905 (2008); 10.1063/1.3029747

Study on microwave emission mechanisms on the basis of hypervelocity impact experiments on various target plates

J. Appl. Phys. 101, 124901 (2007); 10.1063/1.2732401

Microwave emission due to hypervelocity impacts and its correlation with mechanical destruction J. Appl. Phys. 92, 5550 (2002); 10.1063/1.1513885

\section{AIP $\left.\right|_{\text {APL Photonics }}$}

APL Photonics is pleased to announce Benjamin Eggleton as its Editor-in-Chief

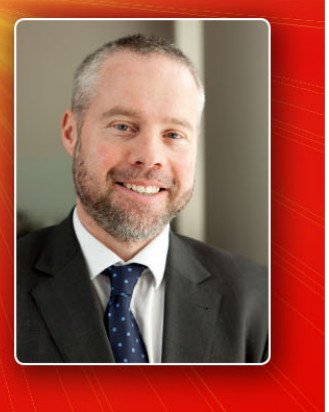




\title{
Examining the temporal evolution of hypervelocity impact phenomena via high-speed imaging and ultraviolet-visible emission spectroscopy
}

\author{
J. D. Tandy, ${ }^{1, a)}$ J. M. Mihaly, ${ }^{2}$ M. A. Adams,${ }^{2}$ and A. J. Rosakis ${ }^{2}$ \\ ${ }_{1}^{1}$ Department of Chemistry, University of Leicester, Leicester LE1 7RH, United Kingdom \\ ${ }^{2}$ Graduate Aerospace Laboratories, California Institute of Technology, Pasadena, California 91125, USA
}

(Received 3 March 2014; accepted 2 July 2014; published online 15 July 2014)

\begin{abstract}
The temporal evolution of a previously observed hypervelocity impact-induced vapor cloud [Mihaly et al., Int. J. Impact Eng. 62, 13 (2013)] was measured by simultaneously recording several full-field, near-IR images of the resulting emission using an $O M A-V$ high-speed camera. A two-stage light-gas gun was used to accelerate $5 \mathrm{mg}$ Nylon $6 / 6$ right-cylinders to speeds between $5 \mathrm{~km} / \mathrm{s}$ and $7 \mathrm{~km} / \mathrm{s}$ to impact $1.5 \mathrm{~mm}$ thick 6061-T6 aluminum target plates. Complementary laserside-lighting [Mihaly et al., Int. J. Impact Eng. 62, 13 (2013); Proc. Eng. 58, 363 (2013)] and frontof-target (without laser illumination) images were also captured using a Cordin ultra-high-speed camera. The rapid expansion of the vapor cloud was observed to contain a bright, emitting exterior, and a darker, optically thick interior. The shape of this phenomenon was also observed to vary considerably between experiments due to extremely high-rate $(>250000 \mathrm{rpm})$ of tumbling of the cylindrical projectiles. Additionally, UV-vis emission spectra were simultaneously recorded to investigate the temporal evolution of the atomic and molecular composition of the up-range, impact-induced vapor plume. A PI-MAX3 high-speed camera coupled to an Acton spectrograph was utilized to capture the UV-vis spectra, which shows an overall peak in emission intensity between approximately 6-10 $\mu$ s after impact trigger, corresponding to an increased quantity of emitting vapor/plasma passing through the spectrometer slit during this time period. The relative intensity of the numerous spectral bands was also observed to vary according to the exposure delay of the camera, indicating that the different atomic/molecular species exhibit a varied temporal evolution during the vapor cloud expansion. Higher resolution spectra yielded additional emission lines/bands that provide further evidence of interaction between fragmented projectile material and the $1 \mathrm{mmHg}$ atmosphere inside the target chamber. A comparison of the data to down-range emission spectra also revealed differences in the relative intensities of the atomic/molecular composition of the observed vapor clouds. (C) 2014 AIP Publishing LLC.
\end{abstract}

[http://dx.doi.org/10.1063/1.4890230]

\section{INTRODUCTION}

Hypervelocity impact experiments have been used extensively in an attempt to mimic the type of phenomena that occur during the extremely high-speed impacts $(5 \mathrm{~km} / \mathrm{s}$ and above) between meteoroids/spacecraft and planetary bodies. $^{3-28}$ The substantial interest in hypervelocity events partly stems from the extremely high temperatures and pressures that occur during impact. Indeed, the complexity of the numerous interacting processes that simultaneously occur during hypervelocity impact (mixed phase flow, rapid cooling, fragmentation, melting, ionization, vaporization, etc.), may yield phenomena capable of producing unique molecular environments. ${ }^{29-31}$

There have, however, been far fewer hypervelocity experiments examining the emission (or "flash") resulting from impacts between meteoroids and spacecraft. ${ }^{8,12,32-38}$ Additional studies of this type would be particularly valuable as the intensity and wavelength of the emission produced by hypervelocity impact events is of significant interest in the

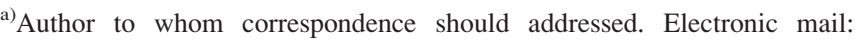
jt245@le.ac.uk
}

engineering and evaluation of spacecraft shielding. In order to effectively protect any light sensitive equipment inside a spacecraft, consideration should be taken to ensure that any external shielding that may be hit during a hypervelocity impact event (from meteoroids or orbital debris) sufficiently minimizes both the physical damage and the resulting flash of light from both the inner surface of the shield and any subsequent debris formed within the craft.

Light-gas guns are probably the most widely used method of generating impact velocities of $>5 \mathrm{~km} / \mathrm{s}$ and have been utilized to examine many aspects of hypervelocity, impact-induced ejecta and debris. ${ }^{1,5,6,8,9,35}$ These facilities have the advantage of replicating an actual impact event, in comparison to other methods (e.g., laser ablation) that attempt to simulate the phenomena formed during hypervelocity impact. Furthermore, hypervelocity impact experiments at velocities consistent with asteroidal impacts $(<25 \mathrm{~km} / \mathrm{s})$ yield distinctly different environments to those formed from laser ablation studies, due to the relatively inefficient interaction between the laser source and solid target. ${ }^{39}$ The use of spectroscopic methods to examine emission from hypervelocity impact phenomena often utilizes intensified high-speed cameras, capable of recording with exposure 
times on the order of $1 \mu \mathrm{s}$ or less. The combination of these cameras with appropriate spectrographic instrumentation may be employed to record both images and emission spectra of the earliest hypervelocity impact-induced ejecta and debris. These observations allow an insight into the atomic and molecular composition of the analyzed phenomena.

The small particle hypervelocity impact range (SPHIR) facility at the California Institute of Technology has several advantages when used to simultaneously record both images emission spectra of hypervelocity impact phenomena. ${ }^{1}$ One advantage of the SPHIR facility is that it uses relatively small impactors $(1.8 \mathrm{~mm}$ diameter $)$ that easily allow the complete ejecta and debris vapor clouds (and other phenomena) to be observed and measured. Larger projectiles (and larger vacuum chambers) are required to fully examine the complete evolution of the ejected vapor plume $e^{5,11,12}$ and, consequently, all SPHIR experiments focus only on the early time $(<1 \mathrm{~ms})$ impact-induced phenomena. Additionally, some other facilities have used metallic projectiles in their studies and measured the resulting atomic/molecular emission of the vapor cloud. This usually leads to relatively simple emission spectra containing a few atomic lines/molecular bands of one or two species. The Nylon $6 / 6$ projectiles utilized in the SPHIR facility yield significantly more diverse emission spectra, due to the relative molecular complexity of the fragmentation products. ${ }^{1}$ Furthermore, the observation of the molecular bands originating from the impactor material may lead to a greater understanding of the decomposition of plastics like nylon under the extreme conditions of hypervelocity impact.

This paper reports our analysis of the temporal evolution of the hypervelocity impact-induced emission of nylon $6 / 6$ projectiles on aluminum targets and examines any potential variation in the observed phenomena given small alterations in the impact velocity. It also describes a preliminary assessment of the variation between up-range and down-range phenomena. For clarification, the term "up-range" refers to all ejecta emanating from the front of the target and moving back in the opposite direction to the initial projectile velocity vector. Similarly, "down-range" refers to material that has either passed through the target or been ejected from the backside of the target in the same direction as the initial impactor. Finally, the paper presents two higher resolution UV-vis spectra to more precisely assign the observed molecular emission.

\section{EXPERIMENTAL}

Near-IR images and UV-vis spectra of several hypervelocity impact events have been recorded using the SPHIR facility in the Graduate Aerospace Laboratories at the California Institute of Technology (GALCIT). The facility consists of a two stage light-gas gun ${ }^{40}$ combined with a variety of different diagnostics to examine several aspects of hypervelocity impact-induced phenomena. ${ }^{1}$ A detailed account of the instrumentation and diagnostic capability of the facility is described elsewhere. ${ }^{1}$ The light gas gun uses compressed hydrogen or helium gas to launch $1.8 \mathrm{~mm}$ diameter nylon $6 / 6$ right-cylinders through a free flight tube into a large evacuated target chamber with an air pressure maintained at approximately $1 \mathrm{mmHg}$. These projectiles are accelerated to impact speeds typically between $5 \mathrm{~km} / \mathrm{s}-7 \mathrm{~km} / \mathrm{s}$ and impact a $150 \mathrm{~mm} \times 150 \mathrm{~mm}$ aluminum target held perpendicular to the shot line of the projectile. ${ }^{1}$ The target thickness may be varied according to the specific experimental aims.

The SPHIR facility diagnostics comprise of: (a) a continuously recording Photron high-speed camera, used to calculate the impactor velocity; (b) an intensified Cordin ultra-high-speed camera, used to record 8 shadowgraph images of the opaque (solid/liquid) ejecta and debris via the described laser-side-lighting (LSL) technique; ${ }^{2}$ (c) a Princeton Instruments near-IR $(0.9 \mu \mathrm{m}$ to $1.7 \mu \mathrm{m})$ highspeed camera, used to record a single image of the emitting impact phenomena, and (d) an intensified Princeton Instruments UV-vis ( $275 \mathrm{~nm}$ to $825 \mathrm{~nm}$ ) high-speed camera coupled to a spectrograph system, used to record a single emission spectrum of either the emitting ejecta or debris.

The near-IR (OMA-V) and UV-vis (PI-MAX3) highspeed camera systems are mounted above the target chamber and view each impact event at a side-profile (parallel to the target), with an angle of approximately $27^{\circ}$ from vertical. ${ }^{1}$ The near-IR camera primarily uses a $25 \mathrm{~mm}$ focal length lens giving a field of view (FOV) of $25.1 \mathrm{~cm} \times 20.0 \mathrm{~cm}$. The UVvis camera's FOV is determined both by the camera lens and the spectrograph slit width (variable) and height (fixed). Typically, a $20 \mathrm{~mm}$ lens is used with a slit width of $100 \mu \mathrm{m}$, yielding a FOV of approximately $1.3 \mathrm{~cm}$ (width) $\times 12.7 \mathrm{~cm}$ (height). The spectral resolution and wavelength coverage in each spectrum were $1.3 \mathrm{~nm}$ and $351 \mathrm{~nm}$, respectively, when utilizing the $150 \mathrm{~g} / \mathrm{mm}$ diffraction grating with a $100 \mu \mathrm{m}$ slit width. The spectrograph slit is primarily positioned in front of the aluminum target to measure the up-range impactinduced emission but was moved briefly behind the target to obtain an emission spectrum of the down-range phenomena. All spectra were calibrated using the Princeton Instruments $\mathrm{Hg} / \mathrm{Ne}-\mathrm{Ar}$ light source and the automated IntelliCal calibration procedure. ${ }^{1}$

More recently, the SPHIR facility has also added a second Cordin ultra-high-speed camera, used to record six additional images of the impact-induced emission from the front of the target, without laser illumination. The camera is positioned to image the front face of the target at an angle of approximately $11^{\circ}$ from horizontal with respect to the projectile shot line. The incorporation of this perspective allows observations of the radial expansion of the emission emanating from all up-range phenomena.

All in-situ diagnostics are triggered simultaneously by an LED photodiode positioned approximately $20 \mathrm{~cm}$ from the target to observe the impact flash generated during each experiment. The relative timing between instrument measurements is therefore precisely known (to within $10 \mathrm{~ns}$ ) and all additional exposure delays are synchronized on this trigger time $\left(t_{\text {trig }}\right)$. From previous work, it is known that the delay between projectile impact and instrument trigger is approximately $3 \mu \mathrm{s} ;^{41}$ this is assumed to remain essentially constant throughout all investigations described herein. 
All diagnostics are used during or after every experiment to provide complementary data of the observed hypervelocity impact phenomena. Although the following results primarily focus on data acquired from the IR to UV-vis camera systems, complementary LSL shadowgraph and front-oftarget images are also presented to provide a clearer interpretation of the observed phenomena.

\section{RESULTS}

The recent description of the SPHIR facility capability ${ }^{1}$ reported examples of the emitting vapor/plasma cloud produced during a hypervelocity impact event at SPHIR, using a $1.8 \mathrm{~mm}$ diameter nylon projectile on an aluminum target at an angle of $0^{\circ}$ from vertical. ${ }^{1}$ The near-IR images of these events showed a large vapor/plasma cloud expanding from the up-range (front) side of the target that contained a bright exterior and dark center. The images also revealed a smaller cloud moving from the back side of the target that represented the emitting vapor that had passed through the target. UV-vis spectra of the hypervelocity impact-induced emission were also reported and showed several molecular bands originating from both the fragmentation of the nylon projectile and the aluminum target.

To more fully understand the nature of this impactinduced vapor/plasma, several aspects have been identified for further investigation. First, the temporal evolution of the up-range vapor cloud has been observed in order to examine the rate of expansion and any discernible changes in the emission intensity or molecular character. The second aspect of this work assesses the effect of impact velocity on the vapor cloud, in order to investigate any significant changes to the phenomena when more (or less) initial kinetic energy is transferred to the target material. Third, certain molecular bands were examined at higher spectral resolution by utilizing the finer $1200 \mathrm{~g} / \mathrm{mm}$ diffraction grating of the PI-MAX3 camera system. Finally, a down-range ejecta emission spectrum was also recorded and compared to the previously measured up-range phenomena.

\section{Preliminary spectra}

In order to select an appropriate spectral range for these studies, two preliminary spectra were recorded across the full range of the PI-MAX3 UV-vis detector. The two spectra were designated shots A and B and ranges of 496-805 nm and 296-605 nm, respectively, were selected. The spectrometer slit width was set at $100 \mu \mathrm{m}$ and positioned $2.5 \mathrm{~cm}$ uprange of the $0.5 \mathrm{~mm}$ thick target. Figure 1 shows the resulting spectra of shots A and B with impact velocities of 5.8 and $6.0 \mathrm{~km} / \mathrm{s}$, respectively. Both shots were recorded using an exposure time of $2.0 \mu \mathrm{s}$ and a delay of $4.3 \mu \mathrm{s}$ after the instrument trigger upon impact. This short time delay allowed the ejecta to expand and sufficiently fill the full FOV of the spectrometer slit.

The spectra in Figure 1 show several strong bands resulting from the atomic and molecular emission of the ejected vapor cloud previously reported. ${ }^{1}$ The preliminary atomic/molecular assignments given to these bands are summarized in Table I and indicate that the emitting species
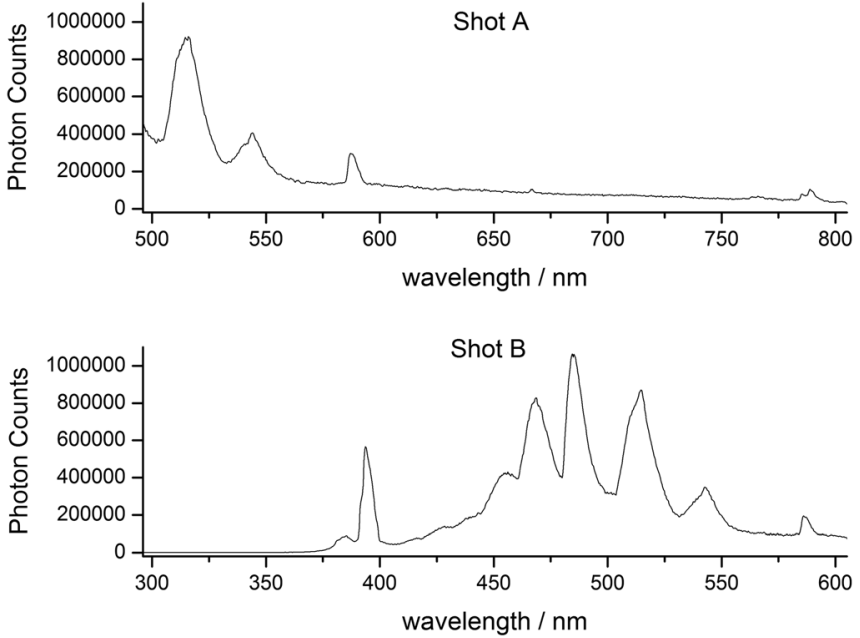

FIG. 1. UV-vis emission spectra of a $1.8 \mathrm{~mm}$ diameter nylon $6 / 6$ projectile impacting a $1.5 \mathrm{~mm}$ thick aluminum target at an angle of $0^{\circ}$ from vertical. The impact velocities of shots A and B were $5.8 \mathrm{~km} / \mathrm{s}$ and $6.0 \mathrm{~km} / \mathrm{s}$, respectively, and the chamber pressures were $1.3 \mathrm{mmHg}$ and $1.2 \mathrm{mmHg}$. Both spectra were recorded using an exposure length $\left(\mathrm{t}_{\mathrm{exp}}\right)$ of $2.0 \mu \mathrm{s}$ and a delay $\left(\mathrm{t}_{\mathrm{del}}\right)$ of $4.3 \mu \mathrm{s}$ after the instrument trigger. The spectrometer slit was positioned approximately $2.5 \mathrm{~cm}$ in front of the target.

originate from both the aluminum target and Nylon 6/6 projectile material. ${ }^{42,43}$ At pressures around $1 \mathrm{mmHg}$, it is possible that the ejected vapor cloud will partially interact with the residual atmosphere, creating oxidized materials (e.g., $\mathrm{AlO})$ that may strongly contribute to the measured emission spectrum. Indeed, $\mathrm{AlO}$ emission is known to dominate the spectra between $450 \mathrm{~nm}$ and $525 \mathrm{~nm},{ }^{12}$ which is clearly evident in Figure 1. The assignments for some of these emission bands are examined more closely in the higher resolution work.

As illustrated in Figure 1, the majority of the atomic and molecular emission occurs between approximately $370 \mathrm{~nm}$ and $600 \mathrm{~nm}$. It was therefore decided that the spectral range chosen in the previously reported spectra (approximately $340 \mathrm{~nm}$ to $650 \mathrm{~nm}$ ) (Ref. 1) would be suitable for the temporal evolution study of the strongest bands.

TABLE I. Preliminary assignments of spectral bands recorded in shots A and B.

\begin{tabular}{lc}
\hline \hline Band head peak $/ \mathrm{nm}$ & Preliminary molecular assignment \\
\hline 386 & $\mathrm{C}_{2}$ \\
389 & $\mathrm{CN} / \mathrm{CH}$ \\
395 & $\mathrm{Al} / \mathrm{O}$ \\
396 & $\mathrm{Al} / \mathrm{O}^{2+}$ \\
456 & $\mathrm{AlC} / \mathrm{CO}^{+}$ \\
465 & $\mathrm{AlO} / \mathrm{C}_{2}$ \\
486 & $\mathrm{AlO}$ \\
512 & $\mathrm{AlO}$ \\
516 & $\mathrm{C}_{2}$ \\
546 & $\mathrm{CO}^{+} / \mathrm{C}_{2}$ \\
589 & $\mathrm{Na}_{2}$ \\
666 & ${\mathrm{O} / \mathrm{NH}_{2}}_{7}$ \\
784 & $\mathrm{~N}_{2}$ \\
789 & $\mathrm{C} / \mathrm{CN}$ \\
\hline
\end{tabular}


TABLE II. UV-vis/IR camera exposure timings and shot conditions for temporal evolution experiments.

\begin{tabular}{|c|c|c|c|c|c|}
\hline Shot ID & Exposure length $\left(\mathrm{t}_{\mathrm{exp}}\right) / \mu \mathrm{s}$ & Exposure delay $\left(\mathrm{t}_{\mathrm{del}}\right) / \mu \mathrm{s}$ & Impactor mass / mg & Impact velocity / km / s & Chamber pressure / $\mathrm{mmHg}$ \\
\hline $\mathrm{T} 1$ & 2.0 & 0.3 & 4.8 & 5.5 & 1.0 \\
\hline $\mathrm{T} 2$ & 2.0 & 2.2 & 5.4 & 5.2 & 1.0 \\
\hline $\mathrm{T} 3$ & 2.0 & 4.3 & 5.8 & 5.7 & 1.1 \\
\hline $\mathrm{T} 4$ & 2.0 & 6.2 & 5.6 & 5.8 & 1.0 \\
\hline $\mathrm{T} 5$ & 2.0 & 8.2 & 5.4 & 5.6 & 1.0 \\
\hline T6 & 2.0 & 10.3 & 5.8 & 5.8 & 1.0 \\
\hline $\mathrm{T} 7$ & 2.0 & 12.2 & 5.6 & 5.4 & 1.2 \\
\hline $\mathrm{T} 8$ & 2.0 & 14.2 & 5.7 & 6.0 & 1.1 \\
\hline T9 & 2.0 & 16.3 & 5.6 & 5.8 & 1.1 \\
\hline $\mathrm{T} 10$ & 2.0 & 18.2 & 5.5 & 5.2 & 1.1 \\
\hline
\end{tabular}

\section{Temporal evolution}

In order to examine the temporal evolution of the hypervelocity impact phenomena, ten near-IR images and UV-vis spectra were recorded in sequential $2 \mu$ s intervals over the first $18 \mu$ s after impact by the nylon $6 / 6$ projectile. A thickness of $1.5 \mathrm{~mm}$ was chosen for the $150 \mathrm{~mm} \times 150 \mathrm{~mm}$ aluminum target and configured perpendicular to the projectile shot line ( $0^{\circ}$ impact obliquity). As both the near-IR and UVvis high-speed cameras are only able to acquire a single image or spectrum of the impact event, several shots were required to complete the study. This requirement inevitably resulted in slight variations in the projectile impact velocities, which were maintained between 5.2 and $6.0 \mathrm{~km} / \mathrm{s}$. The spectrometer slit width of the PI-MAX3 system was set at $100 \mu \mathrm{m}$ and positioned $2.5 \mathrm{~cm}$ up-range of the target. It is important to emphasize that the only contributors to each $\mathrm{UV}$-vis spectrum are the emitting ejecta that pass through the spectrometer slit during the exposure time of the PI$M A X 3$ camera. The resulting spectra therefore illustrate the evolution of the region of space captured within the slit area as the ejected material passes by. Table II shows the projectile velocities and exposure timing of the two cameras

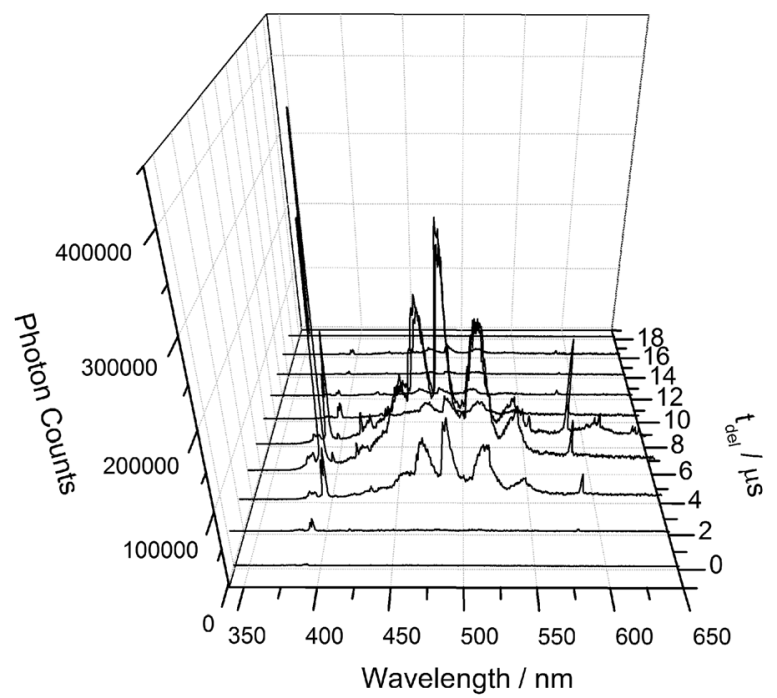

FIG. 2. Ten sequential UV-vis spectra taken with the PI-MAX3 high-speed camera for $1.5 \mathrm{~mm}$ thick targets impacted by $1.8 \mathrm{~mm}$ diameter nylon $6 / 6$ projectiles. Shot conditions and camera exposure timings are given in Table II. (identical for both cameras) for the sequence in addition to the pressure within the target chamber.

Figure 2 shows the resulting UV-vis spectra obtained from the shots listed in Table II. Figure 3 illustrates the same data as a 3D surface to more clearly show the temporal changes in emission intensity across the various spectral bands observed.

In order to further examine the relative temporal behavior of the emission, the five most intense bands observed at approximately $395 \mathrm{~nm}, 465 \mathrm{~nm}, 480 \mathrm{~nm}, 510 \mathrm{~nm}$, and $580 \mathrm{~nm}$ were selected for additional analysis. Figure 4 shows the change in intensity of these bands over the first $20 \mu$ s after impact trigger, as investigated by the shots listed in Table II.

The near-IR images simultaneously recorded during the shots listed in Table II provided a "full-field" view of the near-IR emission arising from both up-range and downrange ejecta. These images are presented in Figure 5 with artificial color added to improve clarity of the emission features and an artificial target overlaid to indicate its position.

Complementary images were also acquired using the LSL technique developed at SPHIR and fully described elsewhere. ${ }^{1,2}$ In order to allow more of the visible impactinduced emission to enter the Cordin camera, the laser power

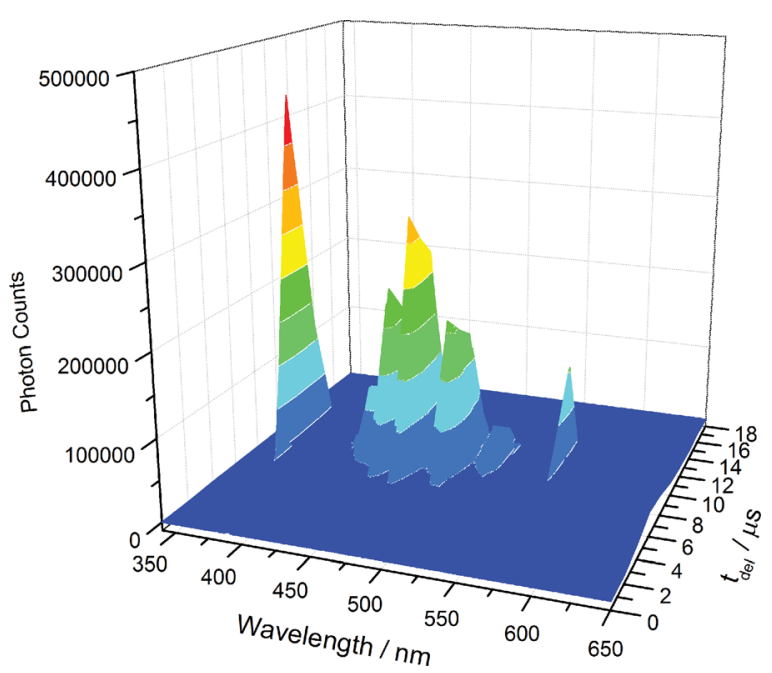

FIG. 3. 3D surface of ten UV-vis spectra taken with the PI-MAX3 highspeed camera for $1.5 \mathrm{~mm}$ thick targets impacted by $1.8 \mathrm{~mm}$ diameter nylon $6 / 6$ projectiles. Shot conditions and camera exposure timings are given in Table II. 


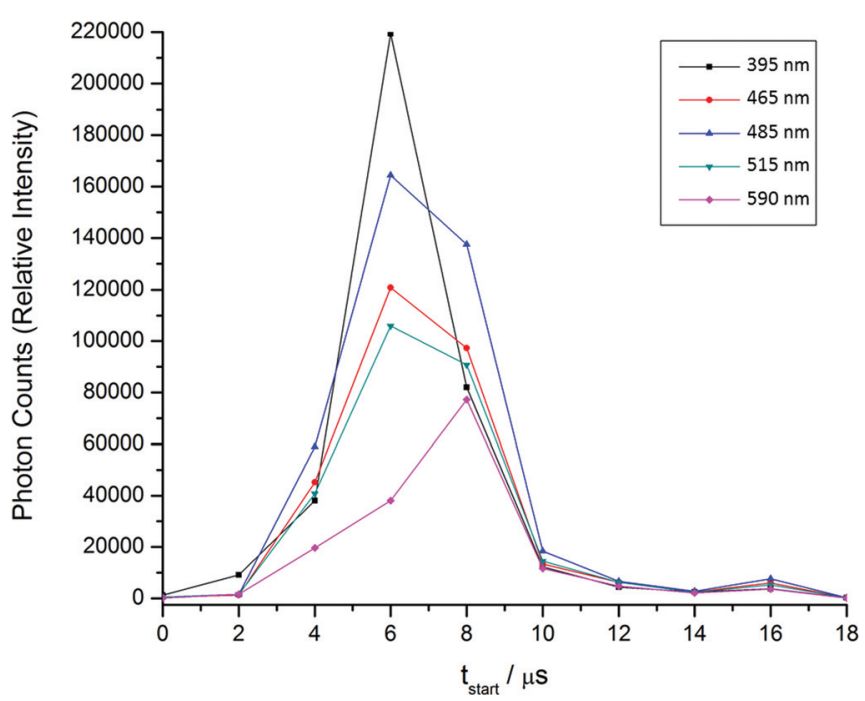

FIG. 4. The relative change in intensity of the five most intense spectral bands observed during the temporal evolution experiments shown in Figure 2.

was reduced from the typical $600 \mathrm{~mW}$ to $60 \mathrm{~mW}$. The LSL images previously published showed distinctly different phenomena to those observed using near-IR imaging. ${ }^{1}$ However, this small alteration to the LSL technique allowed the measurement of similar up-range phenomena to those observed in the near-IR images of Figure 5. Figure 6 illustrates this with a sequence of eight LSL images using $60 \mathrm{~mW}$ laser illumination, taken from a single experiment on a $1.5 \mathrm{~mm}$ thick target impacted at $5.8 \mathrm{~km} / \mathrm{s}$. The FOV of each LSL image is approximately $70 \mathrm{~mm} \times 70 \mathrm{~mm}$ (in comparison to $25.1 \mathrm{~cm} \times 20.0 \mathrm{~cm}$ for the near-IR images) with the exposure timings given in Table III.

Figure 7 shows six additional images that were obtained using the second Cordin camera set at $11^{\circ}$ off the projectile shot line. These front-of-target images simultaneously captured the front view of the target during the same impact used for the LSL results shown previously. As no additional illumination was used for these images (no laser lighting), considerably longer exposure times were required. The FOV of each image is approximately $14 \mathrm{~cm} \times 14 \mathrm{~cm}$ (in comparison to $25.1 \mathrm{~cm} \times 20.0 \mathrm{~cm}$ for the near-IR images) with the exposure timings given in Table IV.

\section{Velocity variation}

The slight variation in shot velocities, whilst exploring the temporal behavior of the emission phenomena, introduces a potentially significant variable into the study. Two additional experiments were therefore carried out to assess the effect of impact velocity on the observed ejecta emission. The impact velocities of the shots were $5.4 \mathrm{~km} / \mathrm{s}$ and $7.0 \mathrm{~km} / \mathrm{s}$ and used identical exposure timing and shot conditions (target thickness, chamber pressure, slit position) to that of shot T6 (Table II) for both the near-IR and UV-vis camera systems. Figures 8 and 9 present the respective near-IR images and $\mathrm{UV}$-vis spectra of the three experiments, varying only the impact velocity.

LSL images were also captured for these three experiments and are illustrated in Figures 6 (impact at $5.8 \mathrm{~km} / \mathrm{s}$ previously shown in the time-evolution study), $10(5.4 \mathrm{~km} / \mathrm{s})$, and $11(7.0 \mathrm{~km} / \mathrm{s})$. The exposure timings of the LSL images in Figures 10 and 11 are given in Table $\mathrm{V}$ and were kept essentially identical as those previously utilized for the images of Figure 6 (Table III).

\section{Higher resolution spectra}

In order to more precisely examine the UV-vis emission of the observed vapor/plasma cloud during the impact experiments, higher resolution spectra were recorded using the finer $1200 \mathrm{~g} / \mathrm{mm}$ diffraction grating of the PI-MAX3 spectrograph. Two distinct spectral regions were chosen for investigation. First, the bands at around $395 \mathrm{~nm}$ (previously designated as primarily aluminum atomic emission) were measured. This spectral region was selected in order observe any potential broadening of the $\mathrm{Al}$ atomic emission lines and to examine whether any other atomic/molecular emission could be identified. Second, the spectral bands between approximately $480 \mathrm{~nm}$ and $520 \mathrm{~nm}$ were examined to see if it was possible to resolve the vibrational structure of the electronic emission bands previously assigned to both $\mathrm{AlO}$ and $\mathrm{C}_{2}$.

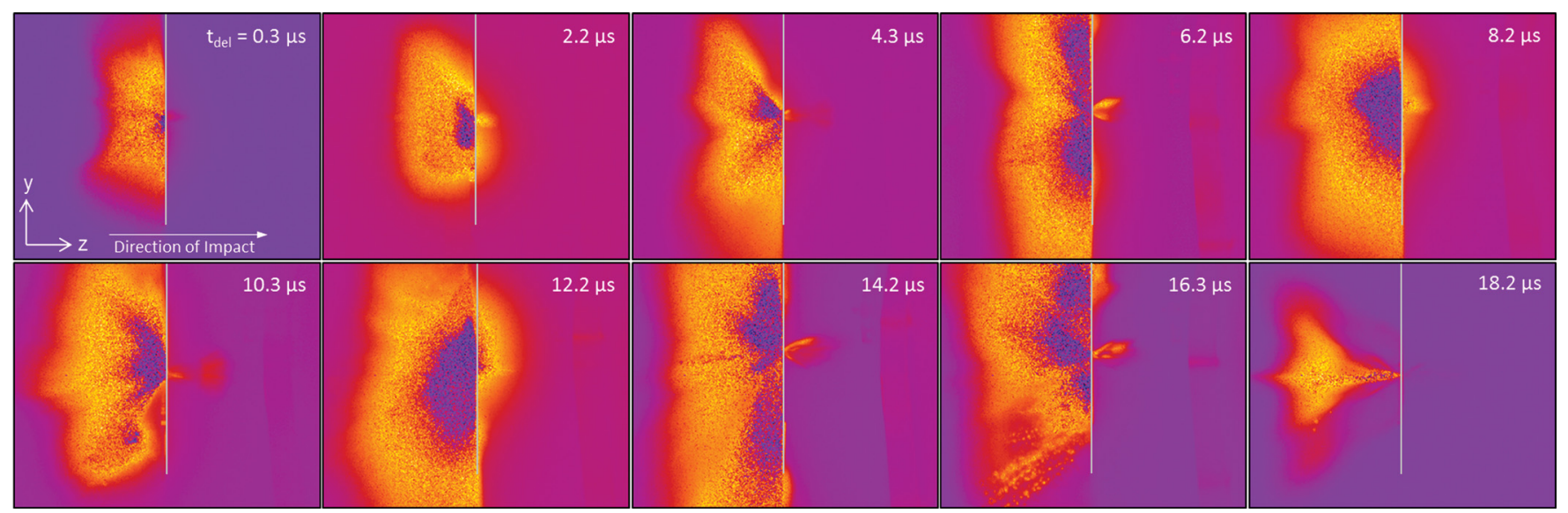

FIG. 5. A sequence of near-IR images taken with the $O M A-V$ high-speed camera for $1.5 \mathrm{~mm}$ thick targets impacted by $1.8 \mathrm{~mm}$ diameter nylon $6 / 6$ projectiles. Shot conditions and camera exposure timings are given in Table II. Artificial color is added to clarify the emission features and an artificial target overlaid to indicate its position. 

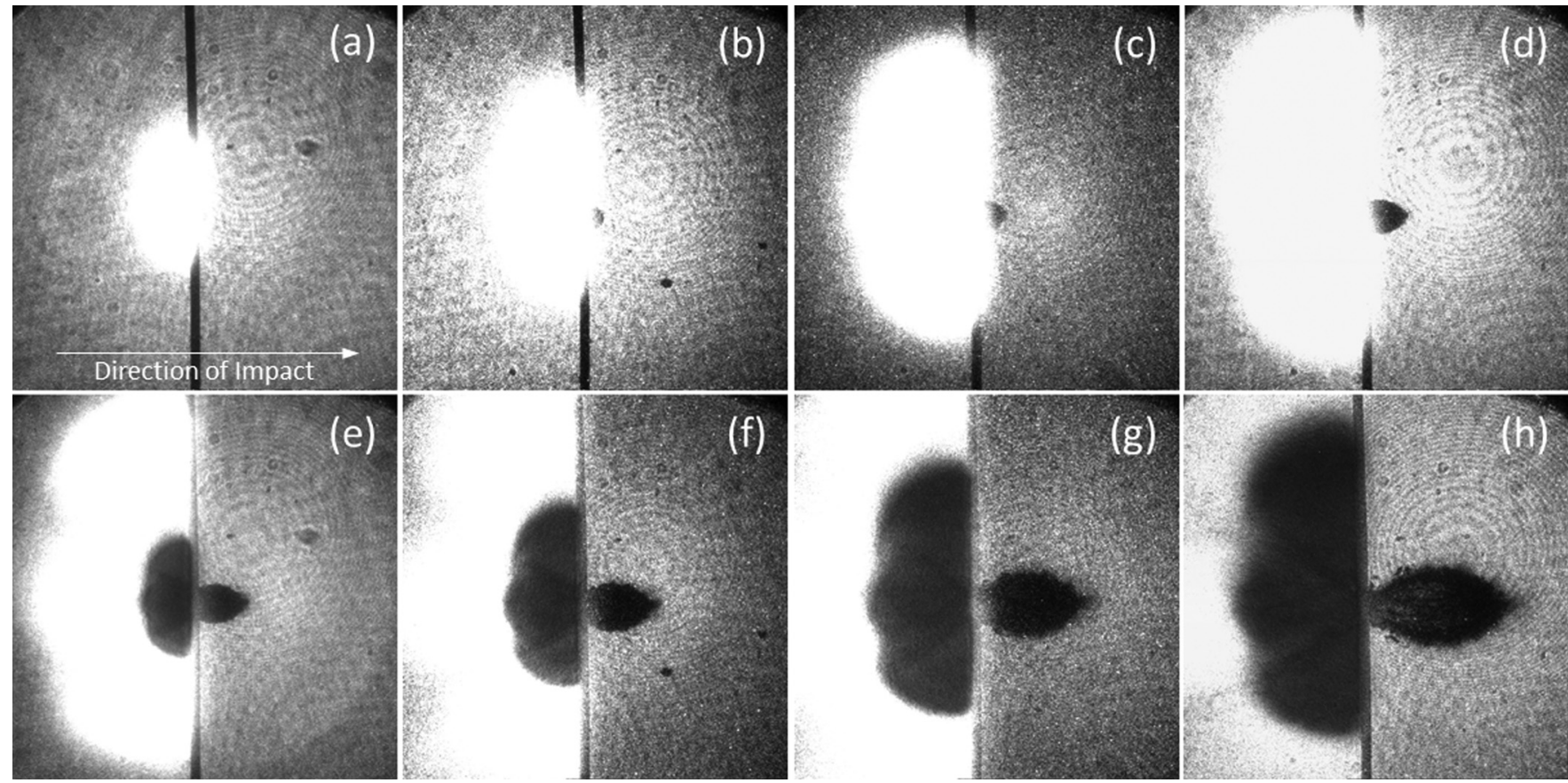

FIG. 6. A sequence of Laser Side-Lighting images taken with a Cordin ultra-high-speed camera for a $1.5 \mathrm{~mm}$ thick target impacted by a $1.8 \mathrm{~mm}$ nylon $6 / 6$ projectile at $5.8 \mathrm{~km} / \mathrm{s}$. The chamber pressure was $1.1 \mathrm{mmHg}$ and exposure timings for each image are given in Table III.

TABLE III. Exposure timings for the LSL images of Figure 6.

\begin{tabular}{lrr}
\hline \hline Figure 6 image & $\mathrm{t}_{\exp } / \mathrm{ns}$ & $\mathrm{t}_{\mathrm{del}} / \mu \mathrm{s}$ \\
\hline (a) & 7.0 & 0.3 \\
(b) & 16.8 & 1.2 \\
(c) & 17.0 & 2.2 \\
(d) & 16.8 & 3.2 \\
(e) & 6.8 & 4.3 \\
(f) & 17.0 & 6.2 \\
(g) & 27.0 & 10.2 \\
(h) & 26.6 & 14.2 \\
\hline \hline
\end{tabular}

Figure 12 shows the higher resolution UV-vis emission spectrum between $371.9 \mathrm{~nm}$ and $408.2 \mathrm{~nm}$ of a $1.5 \mathrm{~mm}$ thick target impacted by a $1.8 \mathrm{~mm}$ diameter nylon $6 / 6$ projectile at $6.0 \mathrm{~km} / \mathrm{s}$. Figure 13 shows the higher resolution UV-vis emission spectrum between $481.4 \mathrm{~nm}$ and $517.7 \mathrm{~nm}$ of a $0.5 \mathrm{~mm}$ thick target impacted by a $1.8 \mathrm{~mm}$ diameter nylon $6 / 6$ projectile at $6.3 \mathrm{~km} / \mathrm{s}$. For both experiments, the chamber pressure was $1.0 \mathrm{mmHg}$ and the $P I-M A X 3$ camera used exposure timings of $\mathrm{t}_{\exp }=5.0 \mu \mathrm{s}$ and $\mathrm{t}_{\mathrm{del}}=0.3 \mu \mathrm{s}$. The spectrometer slit was positioned $2.5 \mathrm{~cm}$ in front of the target. Table VI lists the electronic transitions assigned to the various spectral lines/bands labeled in Figures 12 and 13.
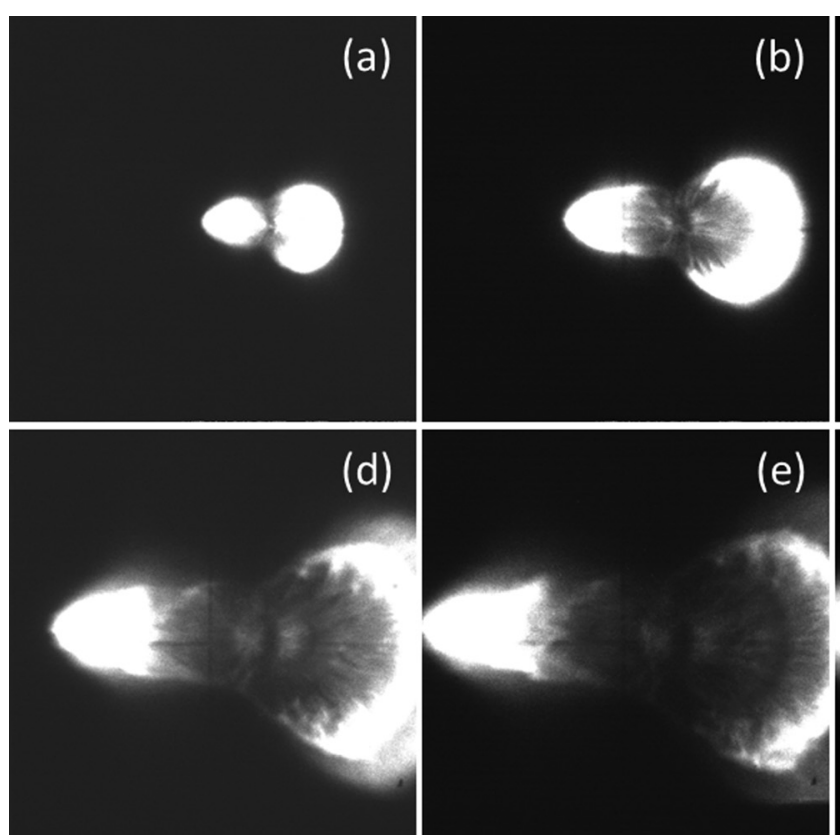

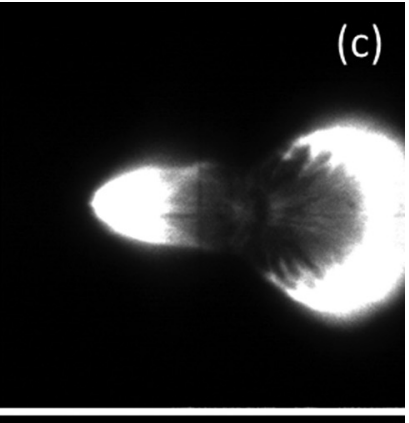

(f)

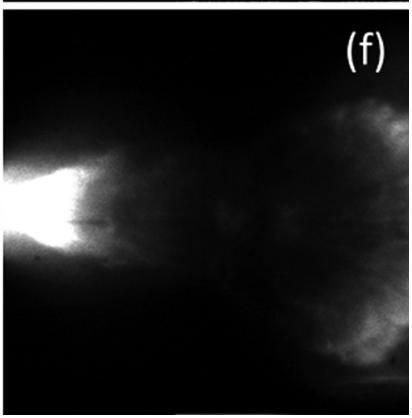

FIG. 7. A sequence of self-illumination images taken with a Cordin ultra-highspeed camera for a $1.5 \mathrm{~mm}$ thick target impacted by a $1.8 \mathrm{~mm}$ nylon $6 / 6$ projectile at $5.8 \mathrm{~km} / \mathrm{s}$. The chamber pressure was $1.1 \mathrm{mmHg}$ and exposure timings for each image are given in Table IV. 
TABLE IV. Exposure timings for the front-of-target images of Figure 7.

\begin{tabular}{lcc}
\hline \hline Figure 7 image & $\mathrm{t}_{\mathrm{exp}} / \mu \mathrm{s}$ & $\mathrm{t}_{\mathrm{del}} / \mu \mathrm{s}$ \\
\hline (a) & 0.8 & 0.1 \\
(b) & 0.8 & 1.7 \\
(c) & 0.8 & 3.3 \\
(d) & 0.8 & 4.9 \\
(e) & 0.8 & 6.5 \\
(f) & 0.8 & 8.1 \\
\hline \hline
\end{tabular}

\section{Down-range ejecta emission}

An analysis of the down-range emission was also undertaken by moving the $100 \mu \mathrm{m}$ PI-MAX3 spectrograph slit $2.5 \mathrm{~cm}$ behind a $1.5 \mathrm{~mm}$ thick aluminum target and recording the resulting UV-vis emission spectrum after impact from a nylon $6 / 6$ projectile. Two shots at impact velocities of 5.7 and $6.6 \mathrm{~km} / \mathrm{s}$ were carried out using a chamber pressure of $1.0 \mathrm{mmHg}$ and an identical camera exposure length $\left(\mathrm{t}_{\mathrm{exp}}\right)$ and delay $\left(\mathrm{t}_{\mathrm{del}}\right)$ of $4.0 \mu \mathrm{s}$ and $0.3 \mu \mathrm{s}$, respectively. Figure 14 shows the two UV-vis emission spectra for these experiments. Figure 15 shows corresponding near-IR images of the same two impacts with identical camera exposure lengths $\left(\mathrm{t}_{\mathrm{exp}}\right)$ and delays $\left(\mathrm{t}_{\mathrm{del}}\right)$ of $1.0 \mu \mathrm{s}$ and $0.3 \mu \mathrm{s}$, respectively.

\section{DISCUSSION}

An inspection of the hypervelocity impact induced emission shown in Figures 2-5 reveal several aspects of its temporal behavior within the initial $20 \mu$ s after impact. Figure 5 shows the gradually expanding, up-range, near-IR emission over the first $20 \mu \mathrm{s}$ after trigger and indicates an expanding phenomenon associated with the impact-induced emission of the vapor cloud. ${ }^{44}$ After an expansion of approximately $10 \mathrm{~cm}$ in the $O M A-V$ camera's FOV, the near-IR emission becomes too weak to be discriminated from the background scattered light observed elsewhere in the image. In addition to the bright expanding emission shown in Figure 5, there is also a smaller and darker region of the expanding phenomena. The outer edges of both the bright and dark areas have almost identical shapes, suggesting the presence of a single up-range phenomenon. The same dark regions are also observed in both the corresponding low-intensity $(6 \mathrm{~mW})$ LSL images and the front-of-target images of Figures 6 and 7 , respectively. The presence of a darker interior suggests that during the vapor cloud expansion, there is a point in time at which the emission ceases or is obstructed by an

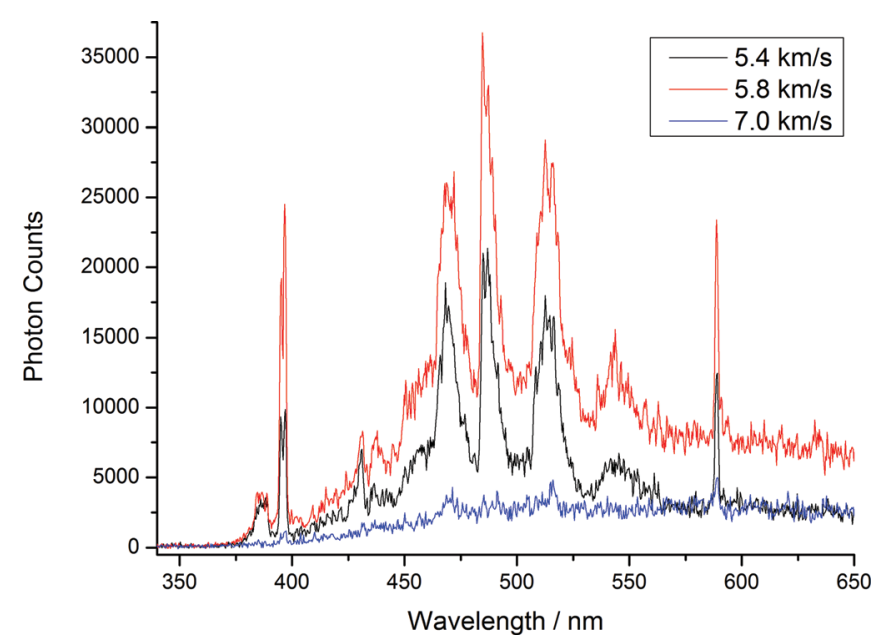

FIG. 9. UV-vis spectra taken with the PI-MAX3 high-speed camera for $1.5 \mathrm{~mm}$ thick targets impacted by $1.8 \mathrm{~mm}$ diameter nylon $6 / 6$ projectiles at $5.4 \mathrm{~km} / \mathrm{s}, 5.8 \mathrm{~km} / \mathrm{s}$ and $7.0 \mathrm{~km} / \mathrm{s}$, respectively. The chamber pressure for each experiment was $1.1 \mathrm{mmHg}$ and the camera used identical exposure timings of $\mathrm{t}_{\exp }=2.0 \mu \mathrm{s}$ and $\mathrm{t}_{\mathrm{del}}=10.3 \mu \mathrm{s}$.

optically thick material between the camera lens and emitting gas/plasma. The latter explanation was previously discounted, as previous higher intensity $\left(76 \mathrm{~W} / \mathrm{m}^{2}\right) \mathrm{LSL}$ photography did not show any obscuration in the vicinity of the observed dark interior in the near-IR images. ${ }^{1}$ However, more recent, lower intensity $\left(7.6 \mathrm{~W} / \mathrm{m}^{2}\right) \mathrm{LSL}$ images (Figure 6) clearly show a darker interior region (of identical shape to the bright region), which obscures the laser light source, indicating that there should be optically thick material present. Furthermore, the corresponding region observed in the near-IR images is noticeably darker than the surrounding background, which also suggests the occurrence of an obscuring or absorbing gas/plasma.

It is clear from the individual shot images in Figure 5 that the near-IR emission formed during the vapor cloud expansion is not hemispherical and contains several irregularities in its size and shape at particular time intervals. This is also supported by the sequence of images in Figure 7 , which show a distinctly asymmetric radial expansion of the visible up-range emission. There are several factors that may cause the inconsistencies in the vapor cloud size, including slight variations in impact velocity, chamber pressure, projectile mass and impact trigger delay. As previously mentioned, the impact trigger delay is assumed to remain essentially constant, although slight variations could
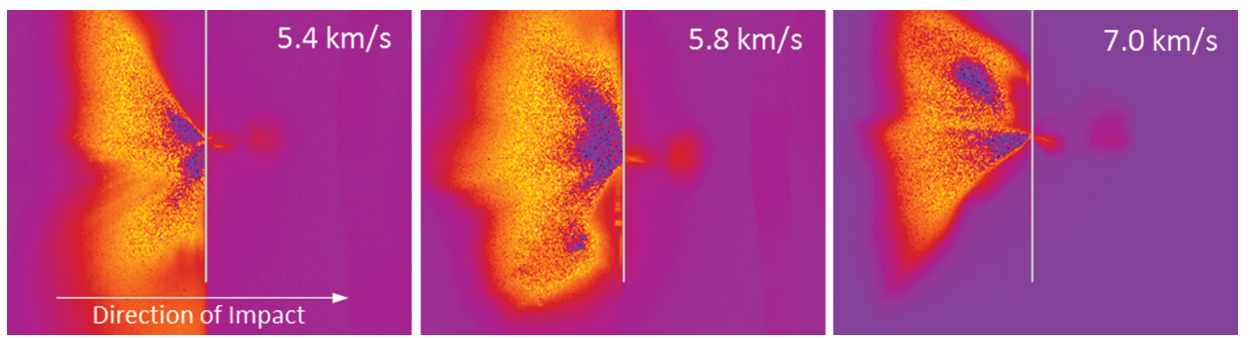

FIG. 8. Near-IR images taken with the $O M A-V$ high-speed camera for $1.5 \mathrm{~mm}$ thick targets impacted by $1.8 \mathrm{~mm}$ diameter nylon $6 / 6$ projectiles at $5.4 \mathrm{~km} / \mathrm{s}$, $5.8 \mathrm{~km} / \mathrm{s}$, and $7.0 \mathrm{~km} / \mathrm{s}$, respectively. The chamber pressure for each experiment was $1.1 \mathrm{mmHg}$ and the camera used identical exposure timings of $\mathrm{t}_{\text {exp }}=2.0 \mu \mathrm{s}$ and $\mathrm{t}_{\mathrm{del}}=10.3 \mu \mathrm{s}$. Artificial color is added to clarify the emission features and an artificial target overlaid to indicate its position. 

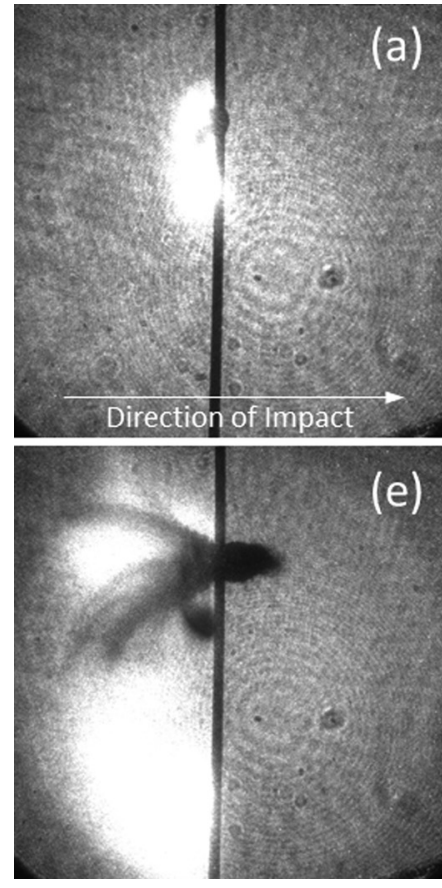

(e)

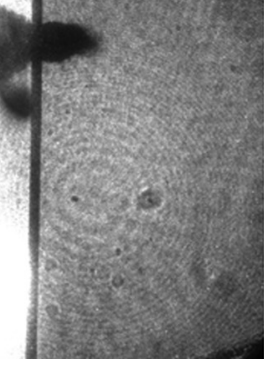

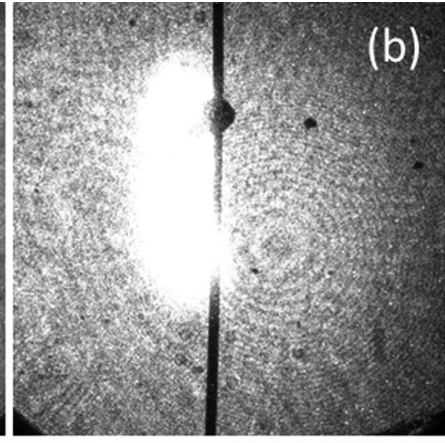

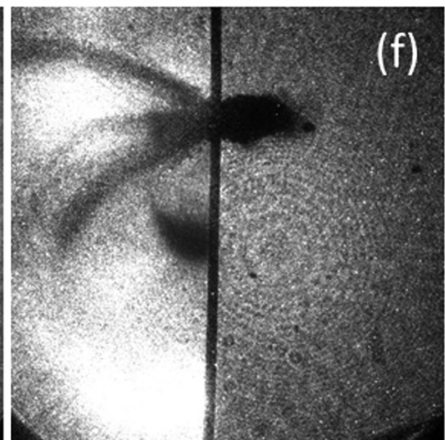

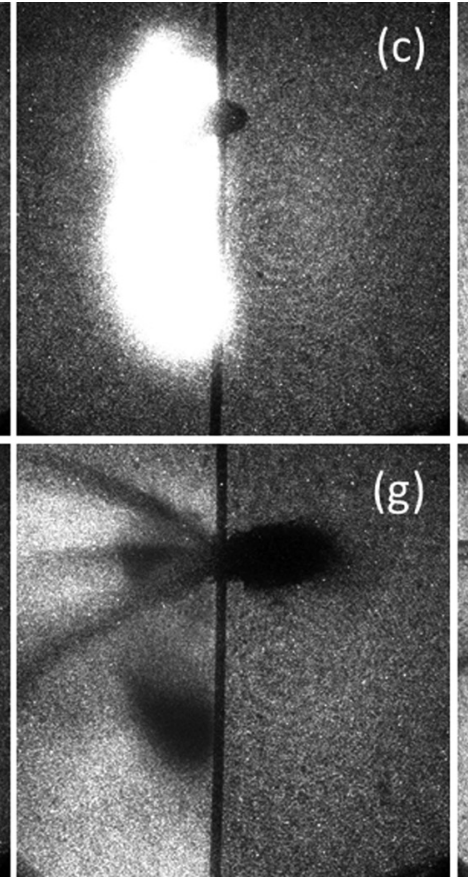

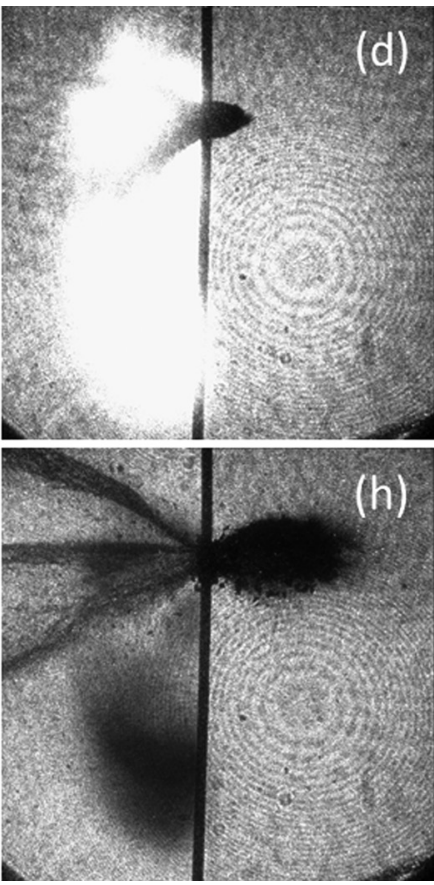

FIG. 10. A sequence of Laser Side-Lighting images taken with a Cordin ultra-high-speed camera for a $1.5 \mathrm{~mm}$ thick target impacted by a $1.8 \mathrm{~mm}$ nylon $6 / 6$ projectile at $5.4 \mathrm{~km} / \mathrm{s}$. The chamber pressure was $1.0 \mathrm{mmHg}$ and exposure timings for each image are given in Table V.

potentially alter the exposure timing of the in-situ instrumentation and, thus, the size of the near-IR emission. The effect of these differences is estimated to be $<1 \mathrm{~mm}$, assuming a maximum trigger delay variance of $0.3 \mu$ s between individual shots. Previous work examining the effect of chamber pressure on the near-IR emission ${ }^{41}$ found that a pressure increase from $0.9 \mathrm{mmHg}$ to $1.1 \mathrm{mmHg}$ decreased the approximate measured radius of the vapor cloud by around $8 \mathrm{~mm}$. Table II shows that during the temporal evolution experiments of this work, the chamber pressure varied between $1.0 \mathrm{mmHg}$ and $1.2 \mathrm{mmHg}$, so a similar change in the size of the near-IR emission to that found in the previous study $^{41}$ is expected.

A potentially larger contributor to changes in the size of the recorded near-IR emission is the impact velocity. Table II shows that the impact velocity varied between $5.2 \mathrm{~km} / \mathrm{s}$ and $6.0 \mathrm{~km} / \mathrm{s}$ during the temporal evolution study. A preliminary assessment of this variation was carried out by
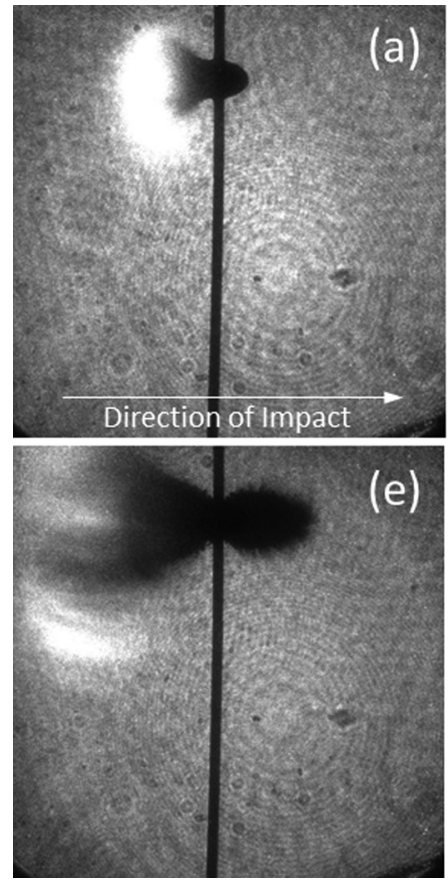
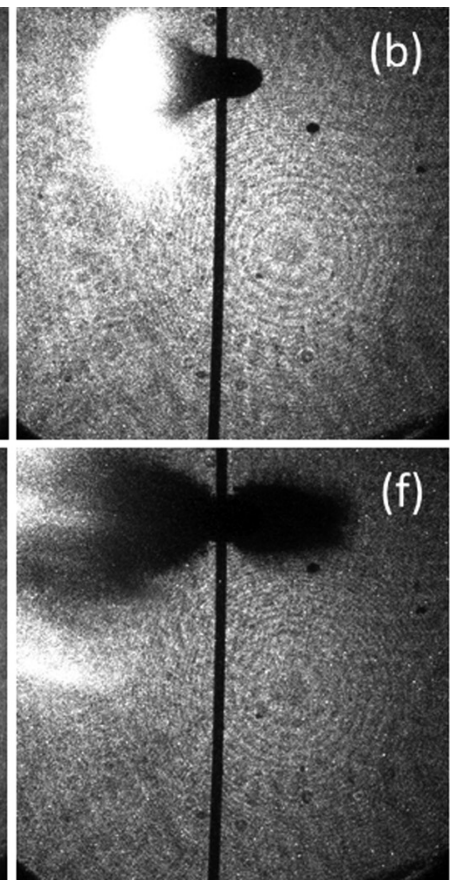
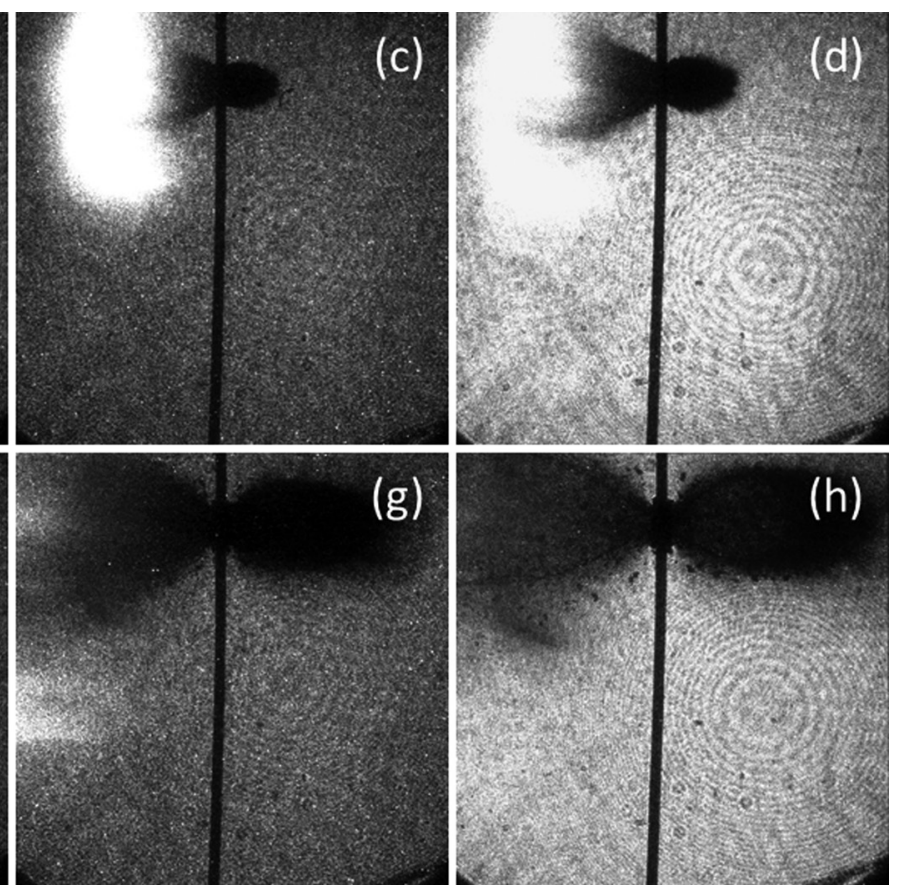

FIG. 11. A sequence of Laser Side-Lighting images taken with a Cordin ultra-high-speed camera for a $1.5 \mathrm{~mm}$ thick target impacted by a $1.8 \mathrm{~mm}$ nylon $6 / 6$ projectile at $7.0 \mathrm{~km} / \mathrm{s}$. The chamber pressure was $1.0 \mathrm{mmHg}$ and exposure timings for each image are given in Table V. 
TABLE V. Exposure timings for the LSL images of Figures 10 and 11.

\begin{tabular}{lrr}
\hline \hline Figure 10/11 image & $\mathrm{t}_{\exp } / \mathrm{ns}$ & $\mathrm{t}_{\mathrm{del}} / \mu \mathrm{s}$ \\
\hline (a) & 7.0 & 0.3 \\
(b) & 16.8 & 1.2 \\
(c) & 17.2 & 2.2 \\
(d) & 16.8 & 3.2 \\
(e) & 6.8 & 4.3 \\
(f) & 16.8 & 6.3 \\
(g) & 27.0 & 10.3 \\
(h) & 26.6 & 14.3 \\
\hline \hline
\end{tabular}

qualitatively analyzing the three near-IR images, illustrated in Figure 8, with impact velocities of $5.4 \mathrm{~km} / \mathrm{s}, 5.8 \mathrm{~km} / \mathrm{s}$, and $7.0 \mathrm{~km} / \mathrm{s}$, respectively, and using identical shot conditions and exposure timings. Ignoring the apparent discrepancies in the shape of the emission (described later), the approximate size of the up-range ejecta is relatively consistent across all three images. As previously discussed, this is probably due to the near-IR emission being indistinguishable from the background scattered light at a vapor cloud expansion radius of $\sim 10 \mathrm{~cm}$. However, the size of the darker, optically thick material does appear to expand slightly with an increase in the initial impact velocity, implying a more rapid growth of the vapor cloud at higher shot velocities. This observation is somewhat supported by the LSL image sequences of Figures 6,10 , and 11 , which also illustrate the vapor cloud expansion at impact velocities of $5.8 \mathrm{~km} / \mathrm{s}, 5.4 \mathrm{~km} / \mathrm{s}$, and $7.0 \mathrm{~km} / \mathrm{s}$, respectively. These images also show a slightly accelerated rate of expansion of the measured visible emission at higher impact velocities, although these observations are blurred by both the change in impact position between shots and the varied angular distribution of the developing ejecta.

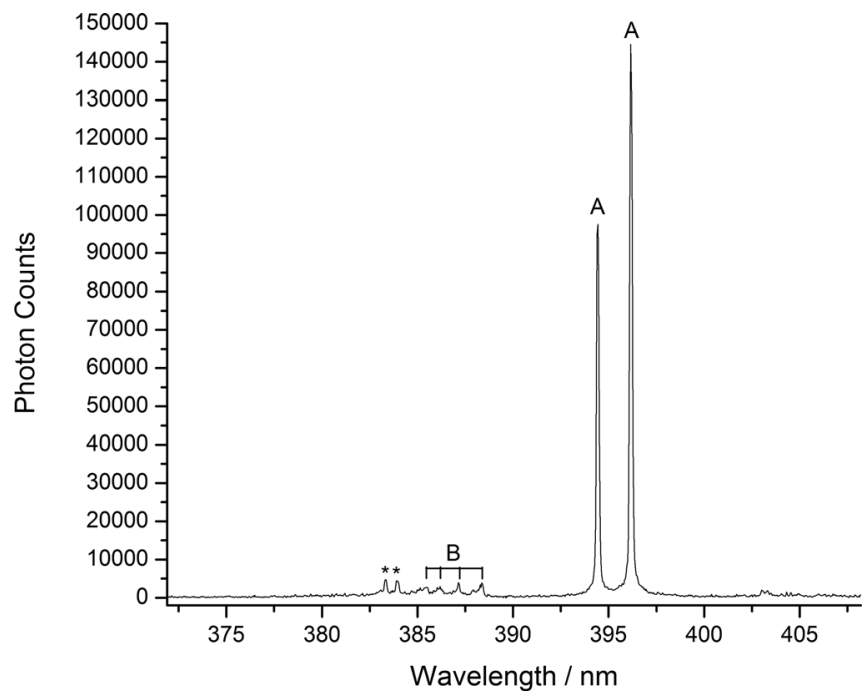

FIG. 12. A higher resolution UV-vis spectrum taken with the PI-MAX3 high-speed camera for a $1.5 \mathrm{~mm}$ thick target impacted by a $1.8 \mathrm{~mm}$ diameter nylon $6 / 6$ projectile at $6.0 \mathrm{~km} / \mathrm{s}$. The chamber pressure was $1.0 \mathrm{mmHg}$ and the camera used exposure timings of $\mathrm{t}_{\exp }=5.0 \mu \mathrm{s}$ and $\mathrm{t}_{\mathrm{del}}=0.3 \mu \mathrm{s}$. The spectrometer slit was positioned approximately $2.5 \mathrm{~cm}$ in front of the target. Spectral lines/bands A and B are labeled according to the assigned atomic/ molecular electronic transitions shown in Table VI.

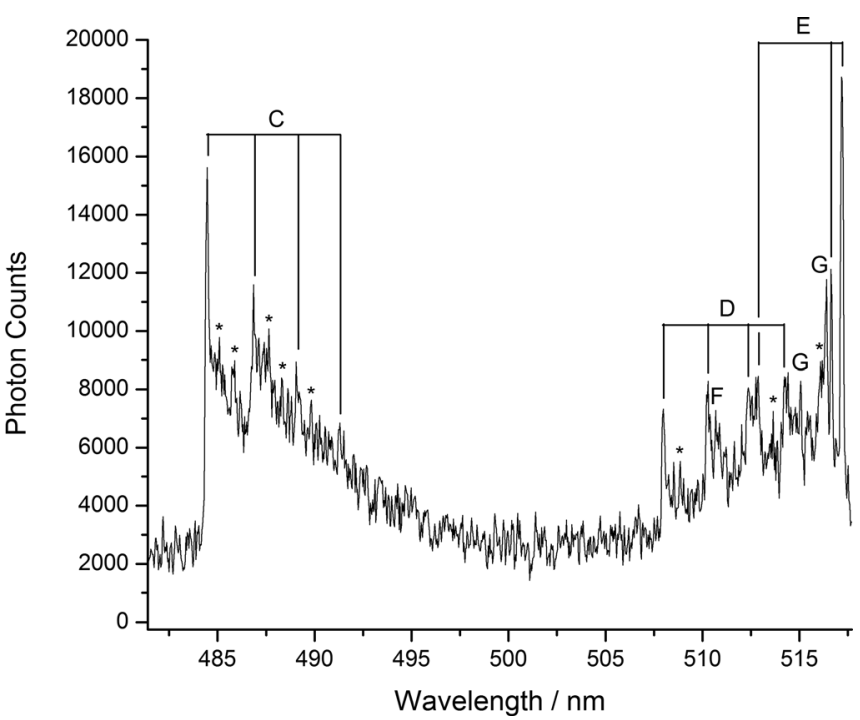

FIG. 13. A higher resolution UV-vis spectrum taken with the PI-MAX3 high-speed camera for a $0.5 \mathrm{~mm}$ thick target impacted by a $1.8 \mathrm{~mm}$ diameter nylon $6 / 6$ projectile at $6.3 \mathrm{~km} / \mathrm{s}$. The chamber pressure was $1.0 \mathrm{mmHg}$ and the camera used exposure timings of $\mathrm{t}_{\exp }=5.0 \mu \mathrm{s}$ and $\mathrm{t}_{\mathrm{del}}=0.3 \mu \mathrm{s}$. The spectrometer slit was positioned approximately $2.5 \mathrm{~cm}$ in front of the target. Spectral lines/bands $\mathrm{C}-\mathrm{G}$ are labeled according to the assigned atomic/ molecular electronic transitions shown in Table VI.

Previous work ${ }^{41}$ used Taylor's blast wave dimensional analysis $^{45}$ to derive Eq. (1), relating the radii of the ejecta front, $R$ to the projectile mass, $m$, impact velocity, $v_{\text {impact }}$, chamber pressure, $P_{\text {atm }}$, and time after impact, $t$

$$
R(t)=C\left(\frac{m v_{\text {impact }}}{P_{\text {atm }}}\right)^{1 / 5} t^{2 / 5}
$$

and

$$
C=3.16 K \alpha^{1 / 5},
$$

where $C$ is a dimensionless constant dependent upon the dimensionless parameters $K$ and $\alpha$. This analysis showed Eq. (1) closely describes the vapor cloud expansion with impact conditions (impact velocity, chamber pressure, etc.) utilized in this work. Taylor's approximation was also previously used to assess the internal energy of impact-generated vapor ${ }^{11}$ and indicated that residual atmospheric pressures of $\sim 1 \mathrm{mmHg}$ were insufficient to decelerate the plume close to the point of impact. Using Eq. (1) and the shot parameters in

TABLE VI. Atomic/molecular electronic transition assignments for the lines/bands of Figures 12 and 13.

\begin{tabular}{lcc}
\hline \hline Line or band & Atomic $/$ molecular species & Electronic transition \\
\hline $\mathrm{A}$ & $\mathrm{Al}$ & ${ }^{2} S-{ }^{2} P$ \\
$\mathrm{~B}$ & $\mathrm{CN}$ & $B^{2} \Sigma_{u}^{+}-X^{2} \Sigma_{g}^{+}$ \\
$\mathrm{C}$ & $\mathrm{AlO}$ & $B^{2} \Sigma^{+}-X^{2} \Sigma^{+}$ \\
$\mathrm{D}$ & $\mathrm{AlO}$ & $B^{2} \Sigma^{+}-X^{2} \Sigma^{+}$ \\
$\mathrm{E}$ & $\mathrm{C} 2$ & $d^{3} \Pi_{g}-a^{3} \Pi_{u}$ \\
$\mathrm{~F}$ & $\mathrm{Al}$ & ${ }^{2} P-{ }^{2} S$ \\
$\mathrm{G}$ & $\mathrm{Al}^{2+}$ & ${ }^{2} G-{ }^{2} F$ \\
$\mathrm{H}$ & $\mathrm{Al}^{2+}$ & ${ }^{2} H-{ }^{2} G$ \\
\hline \hline
\end{tabular}




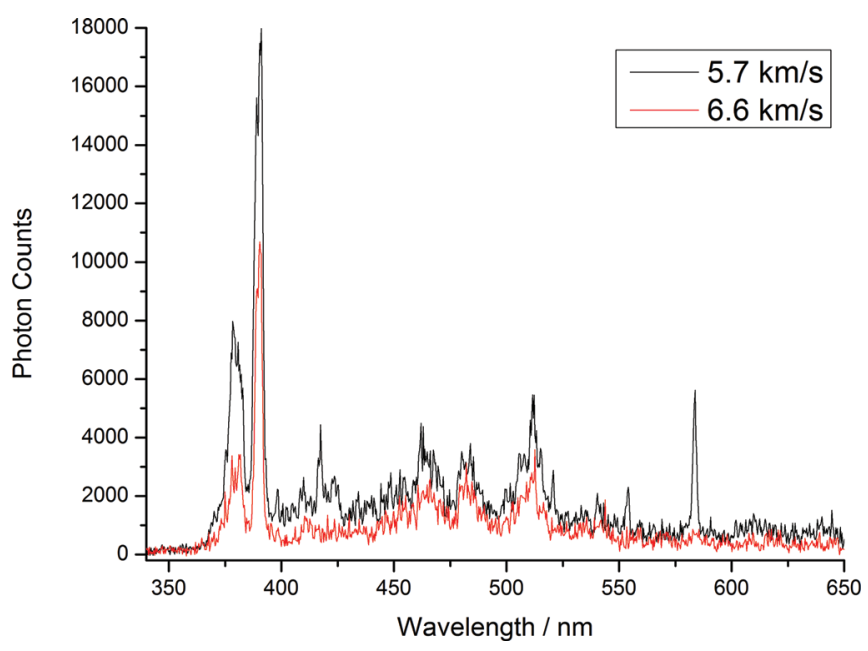

FIG. 14. UV-vis spectra taken with the PI-MAX3 high-speed camera for $1.5 \mathrm{~mm}$ thick targets impacted by $1.8 \mathrm{~mm}$ diameter nylon $6 / 6$ projectiles at $5.7 \mathrm{~km} / \mathrm{s}$ and $6.6 \mathrm{~km} / \mathrm{s}$. The $P I-M A X 3$ spectrometer slit was positioned $2.5 \mathrm{~cm}$ behind the target to record all down-range emission. The chamber pressure for each experiment was $1.0 \mathrm{mmHg}$ and the camera used identical exposure timings of $\mathrm{t}_{\mathrm{exp}}=4.0 \mu \mathrm{s}$ and $\mathrm{t}_{\mathrm{del}}=0.3 \mu \mathrm{s}$.

Table II, the difference in the maximum predicted vapor cloud size at a given time after impact was calculated to be approximately $4.7 \%$. This value suggests that the modest variations in projectile mass and impact velocity are of little consequence given the total uncertainty in the numerous experimental measurements. ${ }^{1,41}$

The variable most likely to cause changes in the irregular shape of the near-IR emission is the projectile orientation upon impact. Indeed, it is known that the nylon 6/6 cylinders tumble at rotational speeds potentially in excess of $250000 \mathrm{rpm}^{1}$ and so any degree of pitch and yaw of the impactor is possible upon initial contact with the aluminum target. This essentially random projectile orientation clearly manifests itself in the shape of measured near-IR emission images of Figure 5. Such dependence of ejecta phenomena on impactor orientation is also supported by the observed disparities in LSL images for repeated experiments, such as those presented in Figures 6, 10, and 11. Further investigation of this phenomenon would be required to ascertain whether the spinning projectile would significantly affect the intensity of the ejecta emission observed in these experiments.

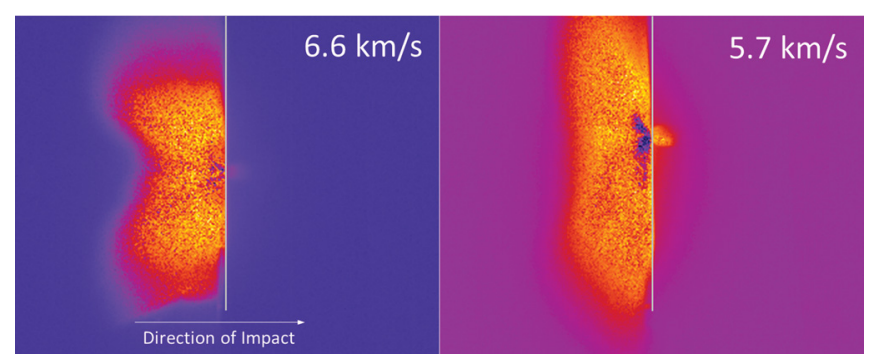

FIG. 15. Near-IR images taken with the $O M A-V$ high-speed camera for $1.5 \mathrm{~mm}$ thick targets impacted by $1.8 \mathrm{~mm}$ diameter nylon $6 / 6$ projectiles at $6.6 \mathrm{~km} / \mathrm{s}$ and $5.7 \mathrm{~km} / \mathrm{s}$, respectively. The chamber pressure for each experiment was $1.0 \mathrm{mmHg}$ and the camera used identical exposure timings of $\mathrm{t}_{\exp }=1.0 \mu \mathrm{s}$ and $\mathrm{t}_{\mathrm{del}}=0.3 \mu \mathrm{s}$. Artificial color is added to clarify the emission features and an artificial target overlaid to indicate its position.
One immediate difficulty arises when comparing the $P I$ $M A X 3 \mathrm{UV}$-vis spectra with both the $O M A-V$ near-IR images and Cordin LSL and front-of-target image sequences. This is of course that the UV-vis spectra are recorded through a narrow slit and do not provide a "full-field" view of the phenomena. Indeed, it is useful to reiterate that the UV-vis spectra only observe emitting ejecta that pass through the spectrometer slit field-of-view. However, given that the distance of the spectrometer slit from the aluminum target is known (approximately $2.5 \mathrm{~cm}$ ), it is possible to show where the UV-vis spectral emission was recorded in relation to the expanding vapor cloud shown in each image. Figure 16 illustrates the UV-vis spectrometer slit position overlaid on LSL images recorded at approximately $2 \mu \mathrm{s}, 6 \mu \mathrm{s}$, and $10 \mu \mathrm{s}$ after impact trigger (previously shown in Figure 6).

The temporal change of the UV-vis spectral emission illustrated in Figures 2-4 shows a relative peak in intensity between approximately $6-10 \mu$ s implying that the maximum quantity of emitting gas/plasma passes through the spectrometer slit FOV during this time period. Figures $2-4$ also show that after approximately $10 \mu \mathrm{s}$, the spectral emission significantly decreases and appears to plateau at $\sim 12 \mu$ s. This observation suggests that at $\sim 10 \mu$ s the darker, optically thick material begins to move through the spectrometer slit and quench the majority of the UV-vis spectral emission. This temporal variation in emitting material passing through the PI-MAX3 spectrometer slit is illustrated in Figure 16. The LSL images first show the spectrometer slit partially filled by emission at $\sim 2 \mu \mathrm{s}$, then, fully filled at $\sim 6 \mu$ s (corresponding to the maximum spectral emission intensity in Figures 2-4) and, then, partially filled by optically thick material at $\sim 10 \mu \mathrm{s}$. The apparent increase in the UV-vis spectral emission intensity to $\sim 6 \mu$ s indicates that the earliest expanding vapor cloud emission shown in the near-IR, LSL, and frontof-target images (Figures 5-7) is not necessarily the most intense. Unfortunately, it is not currently possible to precisely locate a specific region of brightest emission in the near-IR, LSL, or front-of-target images.

Figure 4 clearly shows that not all spectral bands reach their peak intensity at the same time after impact, i.e., the various atomic/molecular species demonstrate a slightly different temporal evolution during the vapor cloud expansion. First, the strongest spectral band at approximately $395 \mathrm{~nm}$ exhibits a sharp increase in emission intensity between $4 \mu \mathrm{s}$

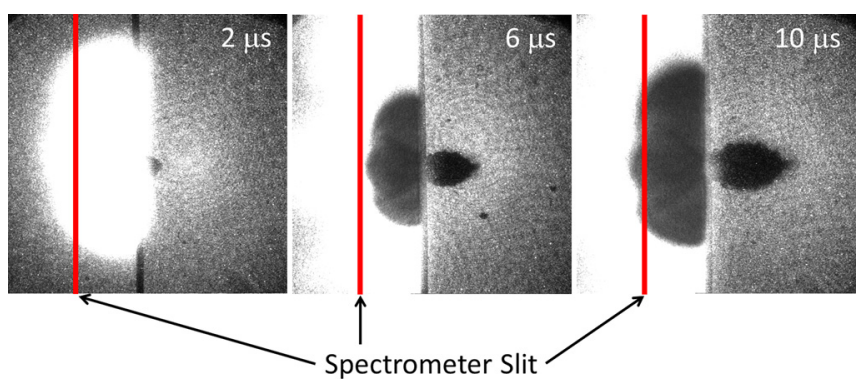

FIG. 16. The approximate field of view of the UV-vis spectrometer slit $(100 \mu \mathrm{m})$ overlaid on LSL images recorded approximately $2 \mu \mathrm{s}, 6 \mu \mathrm{s}$, and $10 \mu$ s after impact trigger. The slit was positioned $2.5 \mathrm{~cm}$ in front of the aluminum target. 
and $6 \mu$ s after impact trigger, followed by a similarly rapid drop in intensity between $6 \mu \mathrm{s}$ and $8 \mu \mathrm{s}$. These observations are perhaps verified by the previous assignment of this band to atomic aluminum emission, which would be expected to be relatively strong and short lived in comparison to the other molecular electronic emission measured. The three spectral bands at approximately $465 \mathrm{~nm}, 485 \mathrm{~nm}$, and $515 \mathrm{~nm}$ show an almost identical temporal evolution, showing a more gradual increase in emission intensity to $\sim 6 \mu \mathrm{s}$, a slight decrease in intensity to $\sim 8 \mu \mathrm{s}$, and a more significant drop to $\sim 10 \mu \mathrm{s}$. These findings are also somewhat in agreement with the previous assignments to the relatively less intense and longer-lived molecular emission of $\mathrm{AlO}$ and $\mathrm{C}_{2}$. Finally, the spectral band at approximately $590 \mathrm{~nm}$ shows an even more gradual increase in emission intensity to its maximum at $\sim 8 \mu \mathrm{s}$, followed by the same swift fall in intensity to $\sim 10 \mu \mathrm{s}$ observed for the other examined spectral bands. This band is assigned to atomic $\mathrm{Na}$ emission, which is often observed as an impurity in hypervelocity impact experiments. The relatively late peak in intensity of this spectral band indicates that the majority of the $\mathrm{Na}$ impurity either travelled more slowly when ejected from the target, or arrived at the spectrometer slit after the nylon impactor after being fired from the gun muzzle.

The higher resolution spectra in Figures 12 and 13 provide additional information regarding the molecular assignments previously shown in Table I. First, Figure 12 confirms the presence of the two $\mathrm{Al}$ atomic lines at $394.4 \mathrm{~nm}$ and $396.2 \mathrm{~nm}$ (labelled A) corresponding to the ${ }^{2} S_{1 / 2}-{ }^{2} P_{1 / 2}$ and ${ }^{2} S_{1 / 2}-{ }^{2} P_{3 / 2}$ transitions, respectively. ${ }^{46}$ These lines do not appear to be significantly broadened by the conditions within the vapor cloud, as their linewidth $(\sim 0.16 \mathrm{~nm}$ FWHM) essentially matches the $0.15 \mathrm{~nm}$ spectral resolution of the $P I$ MAX3 spectrometer system utilizing the $1200 \mathrm{~g} / \mathrm{mm}$ diffraction grating. This indicates that the vapor cloud is relatively diffuse after an expansion of $2.5 \mathrm{~cm}$ to the spectrometer slit. Two additional $\mathrm{Al}$ emission lines were also observed at $510.7 \mathrm{~nm}$, as shown in Figure 13 (labelled F). These lines correspond to the ${ }^{2} P_{1 / 2}-{ }^{2} S_{1 / 2}$ and ${ }^{2} P_{3 / 2}-{ }^{2} S_{1 / 2}$ transitions of atomic aluminum, ${ }^{46}$ which, due to their small separation in wavelength $(0.04 \mathrm{~nm})$, were not able to be resolved in the spectrum.

The emission bands labelled B in Figure 12 correspond to the $(0,0),(1,1),(2,2)$, and $(3,3)$ vibrational progression of the $B^{2} \Sigma_{u}^{+}-X^{2} \Sigma_{g}^{+}$electronic transition of the CN molecule. ${ }^{43}$ The presence of these bands indicates that high vibrational levels of nylon 6/6 fragments are formed and remain populated during the first few microseconds of the vapor cloud expansion. Two sets of high vibrational transitions were also observed in the AlO emission bands illustrated in Figure 13. The sequences labelled $\mathrm{C}$ and $\mathrm{D}$ correspond to the $(0,0),(1,1),(2,2)$, and $(3,3)$ and the $(0,1),(1,2),(2,3)$, and $(3,4)$ vibrational progressions of the $B^{2} \Sigma^{+}-X^{2} \Sigma^{+}$electronic transition of $\mathrm{AlO}$, respectively. ${ }^{43}$ Given the relative strength of these emission bands and the relatively high chamber pressure during the experiments $(\sim 1 \mathrm{mmHg})$, it seems likely that aluminum oxidation occurs during the rapid expansion of the vapor cloud, yielding the relatively strong $\mathrm{AlO}$ emission observed. Additionally, there is evidence for the fragmentation of $\mathrm{AlO}$, due to the appearance of $\mathrm{Al}^{2+}$ ${ }^{2} G-{ }^{2} F$ and ${ }^{2} H-{ }^{2} G$ emission lines at $515.1 \mathrm{~nm}$ and $516.4 \mathrm{~nm}$, respectively (labelled $\mathrm{G}$ and $\mathrm{H}$ ) in Figure $13 .{ }^{46}$ Given the fragmentation of $\mathrm{AlO}$ immediately yields $\mathrm{Al}^{2+}$ (in the correct oxidation state), it is most likely the origin of the two emission lines.

The bands labelled $\mathrm{E}$ in Figure 13 are assigned to the $(0,0)$ and $(1,1) d^{3} \Pi_{g}-a^{3} \Pi_{u}$ transitions (commonly known as the Swan system) of the $\mathrm{C}_{2}$ molecule. ${ }^{43}$ These Swan bands are well known in carbon-rich plasma spectra originating from several sources, including high-speed impacts, the ablation of graphite, the electrical discharge of acetylene, or chemical vapor deposition. ${ }^{8,10,47-51}$ Schultz et al. previously examined spectral signatures from oblique impacts into porous particulates and found evidence that Swan band emission can originate from hydrocarbon bearing targets or from the dissociation of carbon-rich compounds under low atmospheric pressure conditions. ${ }^{10}$ Additional work by Sugita and Schultz investigated impacts of polycarbonate on water and yielded strong $\mathrm{C}_{2}$ Swan band emission, which they attributed to a high-temperature carbon-rich vapor that was ablated from rapidly moving, fine-grain fragments in the expanding impact-induced vapor cloud. ${ }^{8}$ Given the relatively high chamber pressure utilized in these experiments, it is entirely possible that a similar process occurs with the nylon $6 / 6$ projectiles.

A study by Badie et al., examining the solar-induced fluorescence (SIF) of $\mathrm{C}_{2}$, observed a double band head for the $(0,0)$ Swan band transition, with the most intense head at $\sim 517.2 \mathrm{~nm}$ and a significantly weaker secondary band head at the more commonly observed $\sim 516.5 \mathrm{~nm} .{ }^{51}$ Figure 13 shows a similar observation in the $(0,0)$ Swan bands (labelled E) of the impact-induced emission spectra, although the relative intensities of the two band heads are more similar to each other. The SIF emission spectra of Badie et al. were recorded with a graphite target surface temperature of $\sim 3000 \mathrm{~K} .{ }^{51}$ This suggests that a high temperature may be required in order to observe the two $(0,0)$ band heads of the $\mathrm{C}_{2}$ Swan system and that the environment in the vicinity of the hypervelocity impacts in this work may approximately mimic this condition.

Figures 12 and 13 also show several other weak bands that are currently unassigned. We believe the emission of these bands do not correspond to: (a) aluminum from the target material, (b) small atomic or molecular fragments from the nylon 6/6 polymer backbone, or (c) molecules/ions associated with the $1 \mathrm{mmHg}$ of residual air in the target chamber. It is possible however that these emission bands arise from larger molecular chains ${ }^{52}$ originating from the decomposition of the nylon 6/6 impactor or any additives/plasticizers included in the polymer. Alternative sources of these emission bands could be other trace metals originating from the 6061 aluminum target material, impurities from the gun muzzle (e.g., residual powder or launch tube material) or unexpected molecular species from additional reactions of target/impactor material and the residual air. This final explanation may be considered reasonable given the relatively high temperatures and pressures in the vicinity of the impact and may yield potentially interesting molecular products. 
As previously discussed, shot to shot inconsistencies in the expansion of the vapor cloud may arise from several variables including chamber pressure, impactor mass, and impact velocity. The relative emission intensity of each individual experiment should also vary according to these differing shot conditions and so a brief discussion of their influence is appropriate. Although difficult to quantify without significant further work, the effect of chamber pressure is likely to have a minor effect on the emission intensity of the temporal evolution experiments, given the modest change in the vapor cloud expansion (as previously discussed). Several previous studies have found the measured emission intensity, $I$, of an impact-induced flash varies according to impactor mass, $m$, and shot velocity, $v .^{9,32,33,44,53}$ Studies by Eichorn showed that

$$
I=\mathrm{cm}^{1.25} v^{a},
$$

where the constant, $c$, and exponent, $a$, are dependent upon the target and impactor material. ${ }^{32}$ In general, the value of $a$ has been found to vary between 2 and 8, although one study examining carbon impacts on a gold target showed a more complicated behavior at velocities above $5 \mathrm{~km} / \mathrm{s}^{33}$ A closer examination of their data reveals that the emission intensity at impacts between $5 \mathrm{~km} / \mathrm{s}$ and $6 \mathrm{~km} / \mathrm{s}$ (the velocity range of the temporal emission study) stays essentially identical. As carbon is the closest material to nylon 6/6 (essentially a long hydrocarbon structure) utilized during these studies, this indicates that only a relatively small change in emission intensity may be expected due to the variation in the impact velocities during the temporal evolution study. Furthermore, the carbon impacts on gold showed evidence that the emission intensity decreased at impact velocities above $6.5 \mathrm{~km} / \mathrm{s}^{33}$ The impact-induced emission shown in Figures 8,9 , and 11 during the $7.0 \mathrm{~km} / \mathrm{s}$ impact experiment also indicates a reduction in the flash intensity at this velocity and further illustrates the potential similarities in the emission signature of these impactor materials.

Using the relationship, $I \propto m^{1.25}$ from Eq. (3), the variation in intensity due to impactor mass of shots T2-T10 (very little emission was observed in the T1 spectrum) was calculated to be $\sim 8 \%$. Another potential source of impactor mass variation for shot $\mathrm{T} 6\left(\mathrm{t}_{\mathrm{del}}=10 \mu \mathrm{s}\right)$ is also apparent in the near-IR image of Figure 5, which shows a smaller secondary impact below the initial impact. This is most likely caused by a small piece of the nylon impactor breaking off during the free flight before impact and suggests that without the loss of impactor mass, a slightly less abrupt drop in the UVvis emission at $10 \mu$ s (Figure 4) may have been observed.

It is clear from the comparison of both the near-IR images in Figures 5 and 15, and the UV-vis spectra in Figures 2 and 14, that the overall down-range emission is weaker than that observed in the up-range vapor cloud. Additionally, the down-range UV-vis emission spectra in Figure 14 reveal no new molecular bands to those previously observed in the up-range emission, but do display differences in the relative intensities of these bands. Most obvious are the relatively weak bands at approximately $465 \mathrm{~nm}, 484 \mathrm{~nm}$, and $513 \mathrm{~nm}$ (corresponding to $\mathrm{AlO}$ and $\mathrm{C}_{2}$ emission) shown in the down-range spectra. This could indicate that less projectile material has moved through the target in comparison to the aluminum fragments emanating from the backside of the target. The apparent reduction in $\mathrm{AlO}$ emission may be due to the fragmented $\mathrm{Al}$ atoms from the backside of the target possessing less internal and/or kinetic energy, resulting in fewer reactions with either $\mathrm{O}_{2}$ in the air or liberated $\mathrm{O}$ atoms from the nylon projectile decomposition. It is also possible that the nylon $6 / 6$ decomposition progresses further when the projectile material moves through the Al target, causing a relatively lower concentration of $\mathrm{C}_{2}$ in the debris cloud. This is partially supported by the relatively strong $\mathrm{CN}$ emission (at $380 \mathrm{~nm}$ ) in the down-range spectrum at $5.7 \mathrm{~km} / \mathrm{s}$ in Figure 14. An increased fragmentation of the polymeric structure would also seem reasonable due to the relatively high pressure in the vicinity of the target perforation, causing an increased number of molecular collisions.

Figure 14 also shows the effect in the down-range UVvis spectra due to a change in impact velocity, with all emission lines/bands appearing significantly weaker at higher velocity $(6.6 \mathrm{~km} / \mathrm{s}$ versus $5.7 \mathrm{~km} / \mathrm{s})$. This is perhaps due to the more rapid expansion of the up-range vapor cloud, causing less of the fragmented aluminum and Nylon material to move through the target once perforation has occurred and yielding less intense down-range emission. This observation is supported by the near-IR images in Figure 15, which also show weaker down-range emission at $6.6 \mathrm{~km} / \mathrm{s}$ in comparison to $5.7 \mathrm{~km} / \mathrm{s}$.

\section{CONCLUSIONS}

Full-field near-IR, LSL, and front-of-target images of a previously reported vapor cloud $^{1}$ reveal a rapid temporal expansion of the phenomena, containing a bright, emitting exterior and a darker, optically thick interior. The nonhemispherical shape of the vapor cloud emission was observed to significantly vary between experiments due to rapid tumbling of the cylindrical projectiles. UV-vis spectra of the up-range, impact-induced vapor cloud revealed an overall peak in emission intensity between approximately 6-10 $\mu$ s after impact trigger, corresponding to an increased quantity of emitting vapor/plasma passing through the spectrometer slit during this time period. Higher resolution spectra offer further evidence of interaction between fragmented projectile materials and the atmosphere inside the target chamber. Variations in the relative intensity of the spectral bands suggest that different atomic/molecular species exhibit a varied temporal evolution during the vapor cloud expansion. Down-range emission spectra also reveal differences in the relative intensities of the atomic/molecular composition of the vapor clouds.

\section{ACKNOWLEDGMENTS}

This material was based upon work supported by the Department of Energy National Nuclear Security Administration under Award No. DE-FC52-08NA28613. The authors would like to thank an anonymous reviewer for their helpful comments. 
${ }^{1}$ J. M. Mihaly, J. D. Tandy, M. A. Adams, and A. J. Rosakis, Int. J. Impact Eng. 62, 13 (2013).

${ }^{2}$ J. M. Mihaly, A. J. Rosakis, M. A. Adams, and J. D. Tandy, Proc. Eng. 58, 363 (2013).

${ }^{3}$ K. Hamano, S. Sugita, T. Kadono, and T. Matsui, in Lunar and Planetary Science Conference (LPSC) (2003), Vol. XXXIV, p. 1647.

${ }^{4}$ M. J. Burchell, M. J. Cole, and P. R. Ratcliff, Icarus 122, 359 (1996).

${ }^{5}$ S. Sugita, P. H. Schultz, and M. A. Adams, J. Geophys. Res. 103(E8), 19427, doi:10.1029/98JE02026 (1998).

${ }^{6}$ S. Sugita and P. H. Schultz, J. Geophys. Res. 104(E12), 30825, doi:10.1029/1999JE001061 (1999).

${ }^{7}$ K. Tsembelis, M. J. Burchell, M. J. Cole, and N. Margaritis, Int. J. Impact Eng. 35, 1368 (2008).

${ }^{8}$ S. Sugita and P. H. Schultz, J. Geophys. Res. 108(E6), 5051, doi:10.1029/ 2002JE001959 (2003).

${ }^{9}$ S. Sugita, P. H. Schultz, and S. Hasegawa, J. Geophys. Res. 108(E12), 5140, doi:10.1029/2003JE002156 (2003).

${ }^{10}$ P. H. Schultz, C. A. Eberhardy, C. M. Ernst, M. F. A'Hearn, J. M. Sunshine, and C. M. Lisse, Icarus 190, 295 (2007).

${ }^{11}$ P. H. Schultz, J. Geophys. Res. 101(E9), 21117, doi:10.1029/96JE02266 (1996).

${ }^{12}$ P. H. Schultz, S. Sugita, C. A. Eberhardy, and C. M. Ernst, Int. J. Impact Eng. 33, 771 (2006).

${ }^{13}$ S. Sugita and P. H. Schultz, in Lunar and Planetary Science Conference (LPSC) (1998), Vol. XXIX, p. 1751.

${ }^{14}$ P. H. Schultz, B. Hermalyn, A. Colaprete, and K. Ennico, Science 330, 468 (2010).

${ }^{15}$ B. Jean and T. L. Rollins, AIAA J. 8, 1742 (1970).

${ }^{16}$ C. M. Ernst and P. H. Schultz, in Lunar and Planetary Science Conference (LPSC) (2003), Vol. XXXIV, p. 2020.

${ }^{17}$ C. M. Ernst and P. H. Schultz, in Lunar and Planetary Science Conference (LPSC) (2004), Vol. XXXV, p. 1721.

${ }^{18}$ C. M. Ernst and P. H. Schultz, Icarus 190, 334 (2007).

${ }^{19}$ P. H. Schultz, C. M. Ernst, M. F. A'Hearn, C. A. Eberhardy, and J. M. Sunshine, in Lunar and Planetary Science Conference (LPSC) (2006), Vol. XXXVII, p. 2294.

${ }^{20}$ S. Sugita and P. H. Schultz, in Lunar and Planetary Science Conference (LPSC) (2000), Vol. XXXI, p. 2029.

${ }^{21}$ M. Ebert, L. Hecht, A. Deutsch, and T. Kenkmann, in Lunar and Planetary Science Conference (LPSC) (2011), Vol. XXXXII, p. 1400.

${ }^{22}$ S. Ohno, S. Sugita, T. Kadono, K. Ishibashi, G. Igarashi, and T. Matsui, in Lunar and Planetary Science Conference (LPSC) (2005), Vol. XXXVI, p. 1794.

${ }^{23}$ B. Hermalyn, P. H. Schultz, and J. T. Heineck, in Lunar and Planetary Science Conference (LPSC) (2009), Vol. XXXX, p. 2492

${ }^{24}$ C. A. Eberhardy and P. H. Schultz, in Lunar and Planetary Science Conference (LPSC) (2003), Vol. XXXIV, p. 2039.

${ }^{25}$ B. Hermalyn, P. H. Schultz, and J. T. Heineck, in Lunar and Planetary Science Conference (LPSC) (2012), Vol. XXXXIII, p. 2022.

${ }^{26}$ P. H. Schultz, M. A. Adams, J. W. Perry, J. D. Goguen, and S. Sugita, in Lunar and Planetary Science Conference (LPSC) (1998), Vol. XXVII, p. 1149.
${ }^{27}$ S. Sugita, P. H. Schultz, and M. A. Adams, in Lunar and Planetary Science Conference (LPSC) (1999), Vol. XXVIII, p. 1306.

${ }^{28}$ C. A. Eberhardy and P. H. Schultz, in Lunar and Planetary Science Conference (LPSC) (2004), Vol. XXXV, p. 1855.

${ }^{29} \mathrm{M}$. Elvis, Harvard-Smithsonian Centre for Astrophysics, personal communication (2012).

${ }^{30}$ L. Bindi, P. J. Steinhardt, N. Yao, and P. J. Lu, Science 324, 1306 (2009).

${ }^{31}$ Y. Guan, L. Bindi, J. M. Eiler, L. Hollister, G. J. MacPherson, P. J. Steinhardt, and N. Yao, in Lunar and Planetary Science Conference (LPSC) (2011), Vol. XXXXII, p. 2648.

${ }^{32}$ G. Eichhorn, Planet. Space Sci. 23, 1519 (1975).

${ }^{33}$ G. Eichhorn, Planet. Space Sci. 24, 771 (1976).

${ }^{34}$ D. Heunoske, M. Schimmerohn, J. Osterholz, and F. Schäfer, Proc. Eng. 58, 624 (2013).

${ }^{35}$ K. Weber, V. Hohler, and A. J. Stilp, Int. J. Impact Eng. 14, 797 (1993).

${ }^{36}$ R. J. Lawrence, W. D. Reinhart, L. C. Chhabildas, and T. F. Thornhill, Int. J. Impact Eng. 33, 353 (2006).

${ }^{37}$ D. H. Ramjaun, M. Shinohara, and I. Kato, in Proceedings of the 4th International Symposium on Impact Engineering, Impact Engineering, and Application Vol. I and II (2001), p. 139.

${ }^{38}$ P. Tsou and D. J. Griffiths, Int. J. Impact Eng. 14, 751 (1993).

${ }^{39}$ S. Sugita, T. Kadono, S. Ohno, K. Hamano, and T. Matsui, in Lunar and Planetary Science Conference (LPSC) (2003), Vol. XXXIV, p. 1573.

${ }^{40}$ D. J. Grosch and J. P. Riegel, in Proceedings of the 1992 Symposium on Hypervelocity Impact (1992)

${ }^{41}$ J. M. Mihaly, J. D. Tandy, A. J. Rosakis, M. A. Adams, and D. Pullin, "Pressure-dependent, IR-emitting Phenomenon in Hypervelocity Impact," J. Appl. Mech. (in press).

${ }^{42}$ K. P. Huber and G. Herzberg, Constants of Diatomic Molecules, Molecular Spectra and Molecular Structure Vol. IV (Van Nostrand Reinhold Company, 1979).

${ }^{43}$ R. W. B. Pearse and A. G. Gaydon, The Identification of Molecular Spectra, 4th ed. (John Wiley \& Sons Inc., Chapman and Hall, 1976).

${ }^{44}$ J. A. Ang, Int. J. Impact Eng. 10, 23 (1990).

${ }^{45}$ G. I. Taylor, Proc. R. Soc. London, Ser. A 201, 159 (1950).

${ }^{46}$ A. Kramida, Y. Ralchenko, J. Reader, and NIST ASD Team (2013), NIST Atomic Spectra Database (version 5.1), see http://physics.nist.gov/asd, National Institute of Standards and Technology, Gaithersburg, MD.

${ }^{47}$ D. Yadav, V. Gupta, and R. K. Thareja, Spectrochim. Acta B 64, 986 (2009).

${ }^{48}$ A. M. Keszler and L. Nemes, J. Mol. Struct. 695, 211 (2004).

${ }^{49}$ A. Tanabashi, T. Hirao, T. Amano, and P. F. Bernath, Astrophys. J. Suppl. Ser. 169, 472 (2007).

${ }^{50}$ A. N. Goyette, J. E. Lawler, L. W. Anderson, D. M. Gruen, T. G. McCauley, D. Zhou, and A. R. Krauss, J. Phys. D: Appl. Phys. 31, 1975 (1998).

${ }^{51}$ J. M. Badie, G. Flamant, T. Guillard, and D. Laplaze, Chem. Phys. Lett. 358, 199 (2002).

${ }^{52}$ P. Thaddeus and M. C. McCarthy, Spectrochim. Acta A 57, 757 (2001).

${ }^{53}$ J. K. Baird, G. R. Hough, and T. R. King, Int. J. Impact Eng. 19, 273 (1997). 